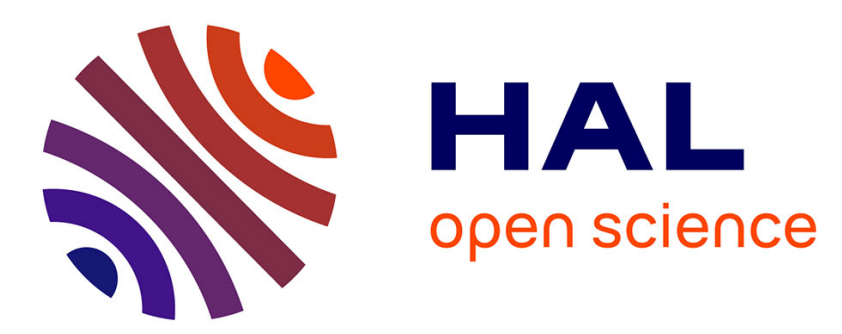

\title{
Some implications of scale relativity theory in avascular stages of growth of solid tumours in the presence of an immune system response
}

C.Gh. Buzea, M. Agop, Evelina Moraru, Bogdan A. Stana, Manuela Gîrţu, Daniela Lancu

\section{To cite this version:}

C.Gh. Buzea, M. Agop, Evelina Moraru, Bogdan A. Stana, Manuela Gîrţu, et al.. Some implications of scale relativity theory in avascular stages of growth of solid tumours in the presence of an immune system response. Journal of Theoretical Biology, 2011, 282 (1), pp.52. 10.1016/j.jtbi.2011.05.001 . hal-00709546

\section{HAL Id: hal-00709546 \\ https://hal.science/hal-00709546}

Submitted on 19 Jun 2012

HAL is a multi-disciplinary open access archive for the deposit and dissemination of scientific research documents, whether they are published or not. The documents may come from teaching and research institutions in France or abroad, or from public or private research centers.
L'archive ouverte pluridisciplinaire HAL, est destinée au dépôt et à la diffusion de documents scientifiques de niveau recherche, publiés ou non, émanant des établissements d'enseignement et de recherche français ou étrangers, des laboratoires publics ou privés. 


\section{Author's Accepted Manuscript}

Some implications of scale relativity theory in avascular stages of growth of solid tumours in the presence of an immune system response

C.Gh. Buzea, M. Agop, Evelina Moraru, Bogdan A. Stana, Manuela Gîrţu, D. Iancu

PII:

S0022-5193(11)00235-9

DOI: doi:10.1016/j.jtbi.2011.05.001

Reference: YJTBI 6465

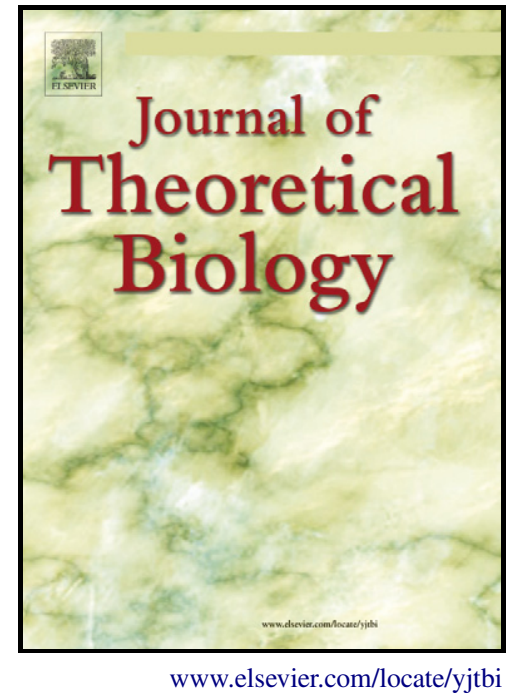

To appear in: $\quad$ Journal of Theoretical Biology

Received date: 12 January 2011

Revised date: $\quad 18$ April 2011

Accepted date: $\quad 3$ May 2011

Cite this article as: C.Gh. Buzea, M. Agop, Evelina Moraru, Bogdan A. Stana, Manuela Gîrţu and D. Iancu, Some implications of scale relativity theory in avascular stages of growth of solid tumours in the presence of an immune system response, Journal of Theoretical Biology, doi:10.1016/j.jtbi.2011.05.001

This is a PDF file of an unedited manuscript that has been accepted for publication. As a service to our customers we are providing this early version of the manuscript. The manuscript will undergo copyediting, typesetting, and review of the resulting galley proof before it is published in its final citable form. Please note that during the production process errors may be discovered which could affect the content, and all legal disclaimers that apply to the journal pertain. 


\title{
Some Implications of Scale Relativity Theory \\ in Avascular Stages of Growth of Solid Tumours \\ in the Presence of an Immune System Response
}

\author{
${ }^{1}$ C. Gh. Buzea, ${ }^{2}$ M. Agop, ${ }^{3}$ Evelina Moraru, ${ }^{3}$ Bogdan A. Stana, ${ }^{4}$ Manuela Gîrțu, ${ }^{3}$ D. Iancu \\ ${ }^{1}$ National Institute of Research and Development for Technical Physics, D. Mangeron 47, \\ Iaşi-700050,Romania; E-mail : calinb2003@yahoo.com \\ 2 “Gh. Asachi” Technical University, D. Mangeron 67, Iaşi - 700050, Romania \\ 3 “Gr. T. Popa” University of Medicine and Pharmacy, University 16, Iaşi-700115, Romania \\ 4,"V. Alecsandri" University, Faculty of Sciences, Calea Mărăşeşti 157, Bacău - 600115, \\ Romania
}

\begin{abstract}
We present a travelling-wave analysis of a reduced mathematical model describing the growth of a solid tumour in the presence of an immune system response in the framework of Scale Relativity theory. Attention is focused upon the attack of tumour cells by tumour infiltrating cytotoxic lymphocytes (TICLs), in a small multicellular tumour, without necrosis and at some stage prior to (tumour-induced) angiogenesis. For a particular choice of parameters, the underlying system of partial differential equations is able to simulate the well-documented phenomenon of cancer dormancy and propagation of a perturbation in the tumor cell concentration by cnoidal modes, by depicting spatially heterogeneous tumour cell distributions that are characterized by a relatively small total number of tumour cells. This behaviour is consistent with several immunomorphological investigations. Moreover, the alteration of certain parameters of the model is enough to induce soliton like modes and soliton packets into the system, which in turn result in tumour invasion in the form of a standard travelling wave. In the same framework of Scale Relativity theory, a very important feature of malignant tumors also results, that even in avascular stages they might propagate and invade healthy tissues, by means of a diffusion on a Newtonian fluid.
\end{abstract}

Keywords : fractal space-time, non-differentiability, cancer, tumor, immune system 


\section{Introduction}

Cancer is a disease derived, with few exceptions, from mutations on single somatic cells that disregard the normal controls of proliferation, invade adjacent normal tissues, and give rise to secondary tumors (metastasis) on sites different from its primary origin [1]. This excessive, autonomous and nonhomeostatic cell growth occurs in multicellular organisms, i.e., animals and plants. In the animal kingdom it was observed even among the most primitive invertebrates of the Arthropoda and Mollusca phyla [2]. Paleopathologists reported neoplasias in dinosaurs whose fossils were dated from around 100 million years. In human beings, authentic cancer-an osteosarcoma-was found in a mandibular fragment of a Pleistocene hominid alive approximately 100 thousand years ago [2].

In the human population, cancer refers to more than 100 forms of a disease that can develop in almost every tissue in the body $[1,2]$. In the United Kingdom, one in four people will die of cancer, whilst one in three will at some point in their life be diagnosed to have cancer (http://www.cancerresearchuk.com/). Of the 135,000 people who died in the Netherlands in $2006,77,000$ (or 57\%) died from a chronic disease, yet cancer was the most frequent cause of death $(40,000)$ [3]. Figures show that $20 \%$ more French men die from cancer each year than British, while Sweden has the EU's lowest male mortality rate from cancer in the EU, with 196 deaths per 100,000 compared to France's 283,3. Cancer is the main cause of premature (before the age of 65) death on the continent, and kills around 960,000 Europeans every year. Moreover, cancer is one of the main causes of morbidity and mortality in the world (http://www.who.org/).

Therefore, developed countries are investing large amounts of money into cancer research in order to find cures and improve existing treatments.

Ninety percent of human cancers are carcinomas, invasive malignant tumors derived from epithelial tissues. A majority of cancers are caused by changes in the cell's DNA because of damage due to the environment. Cancer arises from an interaction of the environment upon the genome. In truth, the double helix structure of the DNA has a limited chemical stability [4] 
and, over time, DNA accumulates damage caused by external mutagens (mutation-causing agents), spontaneous reactions which disintegrate some chemical bonds in DNA under physiological conditions, and errors made during DNA copying in proliferating cells. In Fig. 1, the sites on the DNA strand susceptible to spontaneous intracellular decay are shown. Also, in this figure the main DNA lesions generated by hydrolytic, oxidative, and nonenzymatic methylation processes, as well as the mutations induced by these changes after DNA replication are illustrated. The slow transcription and replication in mammalian cells, which exposes DNA locally in a single-stranded form for longer periods, increases the rates of both hydrolytic depurination and cytosine deamination. So, since the DNA is submitted to millions of replications during the lifespan of an individual, even DNA damage triggered by such natural decay processes has a large chance to occur.

External mutagens include environmental agents (ultraviolet light, ionizing radiation and numerous chemical compounds such as benzo(a)pyrene present in cigarette smoke) and infectious pathogens (the bacterium Helicobacter pylori, more than 100 human papillomaviruses - HPVs, hepatitis-C virus - HCV, Aids virus - HIV, etc.). The damage they create in the genome frequently induces either point mutations affecting single genes or largescale chromosomal mutations which may involve multiple genes [5,6]. Specifically, chemical carcinogens typically cause point mutations in the DNA sequence. Ionizing radiation causes double-strand DNA breaks which presumably lead to chromosomal mutations when incorrectly repaired. Oncogenic viruses introduce exogenous (alien) DNA in normal cells. In Fig. 2 are shown examples of point mutations and translocations which play a major role in cancer formation.

There are two main stages in cancer progression : the initial, relatively benign phase of avascular growth and the subsequent more aggressive vascular phase. Solid tumors in avascular phase are limited in size to no larger than 1-2 $\mathrm{mm}$ in diameter. For a tumor to become metastatic, it needs to be able to nourish the cells at the center of its mass that are too far away 
from blood vessels. This is achieved by angiogenesis, which is a normal process in growth and development, as well as in wound healing. However, this is also a fundamental step in the transition of tumors from a dormant state to a malignant state. It provides a route for the cancer cells to escape into the new blood vessels and circulate throughout the body, where they can deliver to other organs forming metastases. Avascular tumor growth in vivo receives vital nutrients (oxygen, glucose, etc.), and eliminates waste products via diffusive transport [7,8]. Multicellular spheroids formed by transformed cells are widely used as an in vitro avascular tumor models for metastasis and invasion research and for therapeutic screening [9]. The saturated spheroid (106 cells) has a characteristic three-layered structure consisting of an outer rim of nourished dividing cells, an intermediate layer of quiescent cells and a relatively large central necrotic core, where the cells die at a rate which depends on the local nutrient concentration. Dead cells are also found adjacent to both proliferating and quiescent cells [9]. Quiescent cells are said to be in phase $\mathrm{G}_{0}$, in which they are not participating in the cell cycle, but are still viable, metabolically active and they can recover after the restoration of sufficient nutrient [10]. As the spheroid continues to develop, the rim of viable cells at the surface becomes roughly constant in size, leading to a phase of near linear growth $[11,12]$ or later by the combined action of necrotic disintegration, accumulation of waste products [13], mitotic inhibitory factors [14] and cell shedding [15] reduces the rate of tumor growth, leading to a spheroid of a maximal (saturation) size [16-18]. Tumors in vivo continue to develop, due to the mentioned angiogenesis, to sizes over the saturation level [19] and at last become invasive (metastatic), utilizing the body's cavities and vasculature to settle elsewhere, forming secondary growths in the host.

Over the last decade, activity in mathematical modeling and computational simulation of cancer has increased dramatically (e.g., reviews such as [7, 8, 20-23]). A variety of modeling strategies have been developed, each focusing on one or more aspects of cancer. Cellular automata and agent-based modeling, where individual cells are simulated and updated based 
upon a set of biophysical rules, have been developed to study genetic instability, natural selection, carcinogenesis, and interactions of individual cells with each other and the microenvironment. Because these methods are based on a series of rules for each cell, it is simple to translate biological processes (e.g., mutation pathways) into rules for the model. However, these models can be difficult to study analytically, and computational costs can increase rapidly with the number of cells. Because a 1-mm tumor spheroid may have several hundred thousand cells, these methods could become unwieldy when studying tumors of any significant size. See [24-26] for examples of cellular automata modeling, and [27,28] or examples of agent-based modeling. In larger-scale systems where the cancer cell population is on the order of $1,000,000$ or more, continuum methods may provide a more suitable modeling technique. Early work including [29-31] used ordinary differential equations to model cancer as a homogeneous population, as well as partial differential equation models restricted to spherical geometries. Linear and weakly nonlinear analyses have been performed to assess the stability of spherical tumors to asymmetric perturbations [7, 8, 32-35] in order to characterize the degree of aggression. Various interactions of a tumor with the microenvironment, such as stress-induced limitations to growth, have also been studied [36-42]. Most of the modeling has considered single-phase (e.g., single cell species) tumors, although multiphase mixture models have also been developed to provide a more detailed account of tumor heterogeneity [38, 43, 44].

Recently, nonlinear modeling has been performed to study the effects of morphology instabilities on both avascular and vascular solid tumor growth : Cristini et al. (2003) [34] used boundary integral methods to perform the first fully nonlinear simulations of a continuum model of tumor growth in the avascular and vascularized growth stages with arbitrary boundaries. These investigations of the nonlinear regime of shape instabilities predicted encapsulation of external, non-cancerous tissue by morphologically unstable tumors. Li et al. (2007) [35] extended upon [34] in 3-D via an adaptive boundary integral method. Zheng et al. (2005) [45] built upon the model in [34] to include angiogenesis and an extratumoral microenvironment by 
developing and coupling a level set implementation with a hybrid continuum-discrete angiogenesis model originally developed by Anderson \& Chaplain (1998) [46]. As in [34], Zheng et al. found that low-nutrient (e.g., hypoxic) conditions could lead to morphological instability. Their work served as a building block for recent studies of the effect of chemotherapy on tumor growth by Sinek et al. (2004) [47] and for studies of morphological instability and invasion by Cristini et al. (2005) [48] and Frieboes et al. (2006) [49]. Hogea et al. (2006) [50] have also begun exploring tumor growth and angiogenesis using a level set method coupled with a continuum model of angiogenesis. Macklin \& Lowengrub (2007) used a ghost cell/level set method [51] for evolving interfaces to study tumor growth in heterogeneous tissue and further studied tumor growth as a function of the microenvironment [52]. Finally, Wise et al. [53] and Frieboes et al. [54] have developed a diffuse interface implementation of solid tumor growth to study the evolution of multiple tumor cell species, which was employed in Frieboes et al. [55] to model the 3-D vascularized growth of malignant gliomas (brain tumors).

In the case of biological systems the fractal structure of space in which cells interact and differentiate is essential for their self-organization and emergence of the hierarchical network of multiple cross-interacting cells, sensitive to external and internal conditions. Hence, the biological phenomena take place in the space whose dimensions are not represented only by integer numbers $(1,2,3$, etc.) of Euclidean space. In particular, malignant tumors [56-59] and neuronal cells $[60,61]$ grow in a space with non-integer fractal dimension. More precisely, it was proved that the analytical formulae describing the time-dependence of the temporal fractal dimension and scaling factor very well reproduce the growth of the Flexner-Jobling rat's tumor in particular and growth of other rat's tumors in general, also, the results of some test calculations indicated that the formulae derived for the time-dependent temporal fractal dimension and the scaling factor satisfactory describe the experimental data obtained by Schrek for the Brown-Pearce rabbit's tumor growth in the fractal space-time [56-59]. Moreover, the hypothesis that tumorigenesis has a lot in common with the neuronal differentiation and synapse 
formation comes from the fact that they are qualitatively described by the same Gompertz function of growth and take place in the fractal space-time whose mean temporal fractal dimension is lost during progression [60,61].

Since in the vast literature of mathematical modeling and computational simulation of cancer we didn't find any works accounting for the fractal (non-differentiable) nature of the space(-time) itself, where malignant tumors evolve, in this paper we aim to study a model of tumour progression and immune competition by generalized kinetic models, which focus on the development of tumour heterogeneities as a result of tumour cell and macrophage interactions, in the framework of the Scale Relativity Theory.

\section{The Theory of Scale Relativity}

The theory of scale relativity is an extension of the theories of relativity, achieved by applying the principle of relativity not only to motion transformations, but also to scale transformations of the reference system. Recall that, in the formulation of Einstein [62], the principle of relativity consists of requiring that "the laws of nature be valid in every systems of coordinates, whatever their state". Since Galileo, this principle had been applied to the states of position (origin and orientation of axes) and of motion of the system of coordinates (velocity, acceleration). These states are characterized by their relativity, namely, they are never definable in a absolute way. This means that the state of any system (including reference systems) can be defined only relatively to another system.

Laurent Nottale has suggested that the observation scale, i.e., the space and time resolution at which a system is observed or experimented, should also be considered as characterizing the state of reference systems. It is an experimental fact known since Greek philosophers that the scale of a system can be defined only in a relative way: only scale ratios do have a physical meaning, never absolute scales. This may be understood, as briefly explained in what follows. Since the physical parameters we operate with are fractal functions, they will be 
invariant not only on space-time coordinates but on scale ratios, as well. The presence of the scale ratio introduces a new invariance of the physical parameters on dilations and contractions of the space-time operator, which contains a derivative of the type $\partial / \partial \ln \varepsilon$, where $\varepsilon$ is the resolution scale. Since $\varepsilon$ is defined as a space-time scale ratio, we conclude that only scale ratios have physical meaning. As a result, every space-time scale will characterize a physical process associated to a specific complex system (neuronal cells, tumoral cells etc). This led to the proposal that the principle of relativity should be generalized to apply also to the transformations of the scale of reference systems [63-65]. In this new approach, one re-interprets the length and time resolutions, not only as a property of the measuring device and/or of the measured system, but more generally as a property that is intrinsic to the geometry of space-time itself : in other words, space-time is fractal. The principle of relativity of scale then consists of requiring that "'the fundamental laws of nature apply whatever the state of scale of the coordinate system".

\subsection{Motivation}

Let us now consider an essential part of the theory of scale relativity, namely, the description of the effects in standard space-time that are induced by the internal fractal structures of its geodesics. The paper [66] introduced pure scale laws describing the dependence on scale of fractal paths at a given point of space-time. The next step consists of considering a displacement of such a structured point, i.e., the consequences on motion of the non-differentiability. As it was proved, these consequences are radical since they amount to a transformation of Newton's equation of dynamics into a generalized Schrödinger equation.

Note that, in the perspective of potential applications to biological systems, we consider here only the nonrelativistic case (i.e., velocities small with respect to the velocity of light $c$ ). One can show [65] that this case corresponds to a fractal space, while time keeps its regular behaviour. 
As a first step, we shall mainly consider only the simplest case of fractal internal structures, namely, the selfsimilar ones that are characterized by a constant fractal dimension, and more precisely fractal dimension $D_{F}=2$ that plays a critical role in the theory [67]. As we have seen in [66], this behaviour can be derived from a simple, scale-inertial differential equation of first order. It was proved that the laws of mechanics constructed from such internal structures of the geodesics of a fractal space become a quantum-type mechanics. Therefore the various generalizations of internal scale laws that have been considered in [66] naturally lead to generalized quantum laws.

Actually, the discovery that typical quantum mechanical paths (those that contribute mainly to the path integral) are non-differentiable and of fractal dimension 2 is due to Feynman [68], even though the word "fractal"' was coined by Mandelbrot only in [69]. But the various properties of quantum paths described by Feynman in his approach (which is not a return to determinism, since his paths are in infinite number) correspond very closely to the later definition of fractals [70-72]. Now, Feynman derives the fractal and non-differentiable properties of quantum paths from quantum mechanics and its sets of axioms, while the scale relativity approach attempts to do the reverse, namely, found quantum mechanics on the non-differentiable and fractal geometry of space-time.

\subsection{Method}

The method is as follows. We start from a generic description of the displacements in a non-differentiable and continuous space, which is fractal as a consequence, following the fundamental founding theorem of the theory, i.e. Lebesgue's theorem, which states that a curve of finite length is almost everywhere differentiable, while a curve of infinite length is almost everywhere non-differentiable (fractal curve) $[65,67,73,74]$. As we shall see, the paths in a fractal space are characterized by three minimal properties : fractality, infinite number and time irreversibility. 
These three conditions are mathematically expressed at the level of the elementary displacements, then their effect on a physical quantity is described in terms of a "quantumcovariant" derivative. This means that, since the dynamical effects of a space geometry are internal (instead of being external as in the case of an externally added force or field), they are included in the differential calculus itself [62].

In general, in the complex systems (biological systems included), it is difficult to explicitely give all the mathematical factors which intervene in the considered processes. As an example, in plasma physics it is hard to explicitely give the collision terms, the source terms etc. Then, by postulating that motion takes place on continuous and non-differentiable curves, i.e. on fractal curves (we name this approximation - fractal approximation of motion), one can drop all the parameters which describe the clasical motion (e.g., in plasma one can drop notions like collision cross section, mean free path etc.) and consider motion as being free. This method is similar to the one used by Einstein in General Relativity. In such a conjecture, it is necessary to build new differential operators to describe motion, operators which have particular corresponding covariant derivatives. As an example, in Nottale's theory, the covariant derivative corresponding to the motion on Brownian curves (fractal dimension $D_{F}=2$ ) is dependent on a diffusion coefficient specific to the fractal-nonfractal transition. This coefficient has different expressions according to the physical process analysed [65]. Moreover, if an external field exists, e.g. the electromagnetic field, the covariant derivative may contain specific elements of this field, as well, (e.g. the vector potential $\bar{\nabla} \rightarrow \nabla_{\mu}+i g \mathbf{A}$ ) [65].

But in addition to the general (motion) relativity case, in the scale relativity case we have to deal not only with the effects of the geometry, but also with those of the non-differentiability (which does not mean that we cannot define differential elements, but that their ratios, i.e., the derivatives, are sometimes undefined).

Finally, the principle of relativity-equivalence-covariance allows one to write the equation of geodesics as a free form motion equation, which expresses the acceleration 
$\hat{d}^{2} / d t^{2}=0$ in terms of the new covariant derivative $\hat{d}$ (see its construction in what follows). This means that one writes that, locally, there is rectilinear uniform motion, so that all the final complexity comes from the change of reference system itself. The final step amounts to make changes of variables (without any change of the number of degrees of freedom) which transform the classical type of physico-mathematical tool into a quantum-type tool.

\subsubsection{Fractality of the paths}

Strictly, the non-differentiability of the coordinates means that the standard velocity

$$
V(t)=\frac{d X}{d t}=\lim _{d t \rightarrow 0} \frac{X(t+d t)-X(t)}{d t}
$$

is undefined. Namely, when $d t$ tends to zero, either the ratio $d X / d t$ tends to infinity, or it fluctuates without reaching any limit.

However, as recalled above, continuity and non-differentiability imply an explicit dependence on scale (and even a divergence) of the various physical quantities. As a consequence, the coordinate $X(t)$ and the velocity, $V(t)$ are themselves re-defined as explicitly scale-dependent functions $X(t, d t)$ and $V(t, d t)$. We can therefore use again all the arguments developed in [66], and conclude that, in the simplest case, we expect it to be solution of a first order scale differential equation, i.e.,

$$
V(t, d t)=v(t)+w(t, d t)=v(t)\left\{1+\eta(t)\left(\frac{T}{d t}\right)^{1-1 / D_{F}}\right\}
$$

This result means that the velocity is now the sum of two independent terms of different orders of differentiation, since their ratio $v / w$ is, from the standard viewpoint, infinitesimal (see Fig. 3). The $v$ component is what we have called the "classical part" (or differentiable part) of the velocity [75], and $w$ is its "fractal part" (or non-differentiable part). The new component $w$ is an explicitly scale-dependent fractal fluctuation (see Fig. 4) which is described in terms of a dimensionless normalized stochastic variable $\eta(t)$ connected to the type of fractality. In particular, Nottale chooses Wiener-Markov processes $[65,68]$ such that $\langle\eta\rangle=0$ and $\left\langle\eta^{2}\right\rangle=1$. 
As we shall see later on, the final result is totally independent of the probability distribution of this variable.

Here the position on the curve is now located by using the time $t$ itself as a parameter, while the resolution is a time resolution. The scale $T$ must be introduced for dimensional reasons, and appears as a constant of integration. Its presence manifests once again the fact that only scale ratios do have a physical meaning, not the scales themselves.

Eq. (2) multiplied by $d t$ gives the elementary displacement, $d X$, of the system as a sum of two infinitesimal terms of different orders

$$
d X=d x+d \xi
$$

which are such that

$$
\begin{aligned}
& d x=v d t \\
& d \xi=\eta(2 \mathrm{D})^{1-1 / D_{F}} d t^{1 / D_{F}}
\end{aligned}
$$

where the parameter $\mathscr{D}$ is a reformulation of the previous scale $T$ of eq. (2).

Only the critical case of fractal dimension $D_{F}=2$ will be now considered (see $[67,76,77]$ for generalization to a different fractal dimension). The fluctuation becomes

$$
d \xi=\eta \sqrt{2 \mathrm{D}} d t^{1 / 2}
$$

The fundamental parameter $\mathscr{D}$ is bound to play a very important role in what follows. It can be considered to be defined by the above relation, namely,

$$
\mathrm{D}=\frac{1}{2} \frac{\left\langle d \xi^{2}\right\rangle}{d t}
$$

It looks like a coefficient of diffusion, but here its meaning is of geometric essence, namely, it manifests the intrinsic diffusive property of a fractal space, but no external agent or particle is the cause of this "diffusion". This coefficient intervenes in the determination of the fundamental transition from scale dependence (fractality) to scale independence (see Fig. 3). But, as we shall see, it may also be identified, modulo fundamental constants, to a generalization of the Compton scale, $\hbar / m c$, that is the fundamental wavelength which has phenomenologically appeared in 
quantum mechanics (without having, up to now, been theoretically understood from first principles).

\subsubsection{Infinite number of geodesics}

One of the main geometric consequences of the non-differentiability is that there is an infinity of fractal geodesics relating to any couple of points of a fractal space $[65,73]$. This can be easily understood already at the level of fractal surfaces, which can be described in terms of a fractal distribution of conic points of positive and negative infinite curvature [65]. As a consequence, we are led to replace the velocity $V(t, d t)$ on a particular geodesic by the fractal velocity field $V[x(t, d t), t, d t]=v[x(t), t]+w[x(t, d t), t, d t]$ of the whole infinite ensemble of geodesics. This representation is similar to that of fluid mechanics [78], in which the motion of a fluid is described in terms of its velocity field $v(x(t), t)$, its density $\rho(x(t), t)$ and possibly its pressure. We shall indeed recover the fundamental equations of fluid mechanics (Euler and continuity equations), but written in terms of a density of probability (as defined by the set of geodesics) instead of a density of matter, and with an additional term of quantum pressure which occurs as a manifestation of the underlying fractal geometry (see below).

\subsubsection{Discrete symmetry breaking from irreversibility}

A last fundamental consequence of the non-differentiability is the breaking of a discrete symmetry, namely, of the reflection invariance on the differential element of time (it is said to be discrete since it is not a continuous symmetry, such as e.g., translation or rotation, but a discontinuous one such as a mirrored symmetry). It implies a two-valuedness of velocity which can be subsequently shown to be the origin of the fundamental use of complex numbers in quantum mechanics [75]. This use determines a large part of the particularities of quantum mechanics with respect to classical mechanics. 
The derivative with respect to the time $t$ of a differentiable function $f$ can be written twofold [65]

$$
\frac{d f}{d t}=\lim _{\Delta t \uparrow 0} \frac{f(t+\Delta t)-f(t)}{\Delta t}=\lim _{\Delta t \downarrow 0} \frac{f(t)-f(t-\Delta t)}{\Delta t}
$$

The two definitions are equivalent in the differentiable case. In the non-differentiable situation, both definitions fail, since the limits are no longer defined. In the new framework of scale relativity, the physics is related to the behaviour of the function during the zoom operation on the time resolution $\delta t$, identified with the differential element $d t$. The non-differentiable function $f(t)$ is replaced by an explicitly scale-dependent fractal function $f(t, d t)$, which therefore is a function of two variables, $t$ (in space-time) and $d t$ (in scale space). The two functions $f_{+}^{\prime}$ and $f_{-}^{\prime}$ are therefore defined as explicit functions of the two variables $t$ and $d t$

$$
\begin{gathered}
f_{+}^{\prime}(t, d t)=\frac{f(t+d t, d t)-f(t, d t)}{d t} \\
f_{-}^{\prime}(t, d t)=\frac{f(t, d t)-f(t-d t, d t)}{d t}
\end{gathered}
$$

Here we have assumed that $d t>0$. By taking $d t$ algebraic, these two functions would correspond, respectively, to the positive and negative parts of a same unique function. One passes from one definition to the other by the transformation $d t \leftrightarrow-d t$ (differential time reflection invariance), which actually was an implicit discrete symmetry of differentiable physics. When applied to fractal space coordinates $x(t, d t)$, these definitions yield, in the non-differentiable domain, two velocity fields instead of one, that are fractal functions of the resolution, $V_{+}[x(t), t, d t]$ and $V_{-}[x(t), t, d t]$. Each of these fractal velocity field can in turn be decomposed in terms of a classical part and a fractal part, namely, $V_{+}[x(t, d t), t, d t]=v_{+}[x(t), t]+w_{+}[x(t, d t), t, d t]$ and $V_{-}[x(t, d t), t, d t]=v_{-}[x(t), t]+w_{-}[x(t, d t), t, d t]$.

The important fact appearing here is that there is no a priori reason for the two classical parts to be the same. In several works Ord $[79,80]$ also insists on the importance of introducing entwined paths for understanding quantum mechanics (but without giving a cause for this 
fundamental two-valuedness), while Jumarie [81] supports the scale-relativistic view that the use of complex-valued variables appears as a direct consequence of the irreversibility of time.

A simple and natural way to account for this doubling consists of using complex numbers $a+\mathrm{i} b$ and the complex product, according to which $(a+\mathrm{i} b)(c+\mathrm{i} d)=(a c-b d)+\mathrm{i}(a d+b c)$. This is the origin of the complex nature of the wave function of quantum mechanics. Actually, the choice of complex numbers to represent the two-valuedness of the velocity can be proven to be a simplifying and covariant choice $[75,82]$, in the sense of the principle of covariance, according to which the form of the equations of physics should be conserved under all transformations of coordinates. Indeed, the choice of the complex product allows one to suppress what would be additional infinite terms in the final equations of motion.

Another consequence of the combination of the two velocity fields into a single complex velocity field is that, in terms of this physical tool, one recovers a global reversibility of physical laws, as seen from the derivation of the Schrödinger equation.

\subsubsection{Covariant total derivative operator}

We are now led to describe the elementary displacements for both processes, $d X_{ \pm}$, as the sum of a classical part, $d x_{ \pm}=v_{ \pm} d t$, and of a fractal fluctuation $d \xi_{ \pm}$, i.e.,

$$
\begin{aligned}
& d X_{+}(t)=v_{+} d t+d \xi_{+}(t) \\
& d X_{-}(t)=v_{-} d t+d \xi_{-}(t)
\end{aligned}
$$

and similar relations for the other variables. One passes from one process to the other by the transformation $d t \leftrightarrow-d t$. More generally we define two classical derivatives, $d_{+} / d t$ and $\left.d\right\lrcorner d t$, such that

$$
\frac{d_{+}}{d t} x(t)=v_{+}, \quad \frac{d_{-}}{d t} x(t)=v_{-}
$$

These expressions are also valid for three space variables by considering that $x$ and $v$ represent vectors. 
The two derivatives can now be combined to construct a complex derivative operator that allows recovering local differential time reversibility in terms of the new complex process [65]. We define it as

$$
\frac{\hat{d}}{d t}=\frac{1}{2}\left(\frac{d_{+}}{d t}+\frac{d_{-}}{d t}\right)-\frac{i}{2}\left(\frac{d_{+}}{d t}-\frac{d_{-}}{d t}\right)
$$

This choice is motivated by the need to recover, at the classical limit (where the two velocities are equal), the classical real velocity as real part of this complex velocity and a vanishing imaginary part. This is the main tool of the theory.

Applying this operator to the classical part of the position vector yields a complex velocity

$$
\underline{V}=\frac{\hat{d}}{d t} x(t)=\frac{v_{+}+v_{-}}{2}-i \frac{v_{+}-v_{-}}{2}
$$

We call $V$ the real part of this complex velocity and $U$ its imaginary part, i.e. $\underline{V}=V-i U$, with $\mathrm{V}$ $=\left(\mathrm{v}_{+}+\mathrm{v}_{-}\right) / 2$ and $\mathrm{U}=\left(\mathrm{v}_{+}-\mathrm{v}_{-}\right) / 2$.

After having defined the covariant derivative, we now need to find its expression. This will be achieved by explicitly calculating its effect on a given physical quantity.

For this purpose, let us first calculate the derivative of a scalar function $f$. Since the fractal dimension is 2 , we need to go to second order of expansion (this is reminiscent of Einstein's argument about Brownian motion). For one variable it reads

$$
\frac{d f}{d t}=\frac{\partial f}{\partial t}+\frac{\partial f}{\partial X} \frac{d X}{d t}+\frac{1}{2} \frac{\partial^{2} f}{\partial X^{2}} \frac{d X^{2}}{d t}
$$

Once again the generalization of this writing to three dimensions is straightforward [65].

Let us now take the stochastic mean of this expression (i.e., we take the mean of the stochastic variable $\eta$ which appears in the definition of the fractal fluctuation $d \xi$ ). By definition, since $d X=d x+d \xi$ and $\langle d \xi>=0$, we have $<d X>=d x$, so that the second term is reduced (in three dimensions) to $v \cdot \nabla f$. (Recall that this expression denotes the scalar product of the velocity 
$v=\left(v_{x}, v_{y}, v_{z}\right)$ by the gradient $\nabla f=(\partial f / \partial x, \partial f / \partial y, \partial f / \partial z)$ of the function $f$, i.e., in decompacted form, $\left.v \cdot \nabla f=v_{x} \partial f / \partial x+v_{y} \partial f / \partial y+v_{z} \partial f / \partial z\right)$

Now concerning the term $d X^{2} / d t$, it is infinitesimal and therefore not taken into account in the standard differentiable case. But in the non-differentiable case considered here, the mean squared fluctuation is nonvanishing and of order $d t$, namely, $<d \xi^{2}>=2 \mathscr{D} d t$, so that the last term of eq. (15) amounts in three dimensions to a Laplacian (defined as $\Delta=\partial^{2} / \partial x^{2}+\partial^{2} / \partial y^{2}+\partial^{2} / \partial z^{2}$ ). We obtain, respectively, for the (+) and (-) processes,

$$
\frac{d_{ \pm} f}{d t}=\left(\frac{\partial}{\partial t}+v_{ \pm} \cdot \nabla \pm \mathrm{D} \Delta\right) f
$$

The last step consists of recombining the two derivatives into the complex covariant derivative. Substituting eqs. (16) into eq. (13), we finally obtain the expression for the covariant time derivative operator [65]

$$
\frac{\hat{d}}{d t}=\frac{\partial}{\partial t}+\underline{V} \cdot \nabla-i \mathrm{D} \Delta
$$

This is one of the main tools of the theory of scale relativity. Indeed, the passage from standard classical (i.e., almost everywhere differentiable) mechanics to the new non-differentiable theory can now be implemented by replacing the standard time derivative $d / d t$ by the new complex operator $\hat{d} / d t$ [65].

Note that in this replacement, one should remain aware of the fact that this derivative operator is a linear combination of first and second order derivatives, in particular when dealing with the Leibniz rule about derivatives of a product and of composed functions. Now it is possible to build more efficient, fully covariant, tools [83]. This can be made by introducing a velocity operator $\hat{V}=\underline{V}-i \mathrm{D} \nabla$ [84], in terms of which the first order Leibniz rule still applies, since the covariant derivative now reads

$$
\frac{\hat{d}}{d t}=\frac{\partial}{\partial t}+\hat{V} \cdot \nabla
$$


In other words, this means that $\hat{d} / d t$ plays the role of a "covariant derivative operator", namely, we are able, by using it, to write the fundamental equations of physics under the same form they had in the differentiable case.

\section{Avascular Stages of Growth of Solid Tumours in the Presence of an Immune System}

\section{Response}

The immune system is a network of cells, tissues, and organs that work together to defend the body against attacks by foreign bodies. The immune system must recognise foreign bodies to respond. Let's assume that the tumor cells have undergone some changes on the way to malignancy and hence are able to be recognised by the immune system as foreign. In most cases, the immune system destroys foreign bodies in two ways, one is a humoral response which is done by soluble proteins, known as antibodies, in the bodily fluid. The second is a cytotoxic response whereby the killer cells respond to the cancer cells.

For definiteness, let's consider the cytotoxic response. Let $T(r, t)$ be the concentration of the tumor cells at position $r$ and time $t$, and let $E(r, t)$ be the concentration of the effector cells constituting the cytotoxic response. In the absence of immune response, the tumor grows according to the equation

$$
\frac{\partial T}{\partial t}=\rho T\left(1-\frac{T}{K}\right)+D \nabla^{2} T
$$

where $D, K$ and $\rho$ are positive parameters, $D$ being the random motility coefficient of the tumor cells, $K$ the carrying capacity of the environment and $\rho$ the logistic proliferation rate of the tumor cells.

One of the main factors (but not the only one) contributing to the induction and maintenance of cancer dormancy is the reaction of the host immune system to the tumour cells $[85,86]$. Indeed, tumour-associated antigens can be expressed on tumour cells at very early stages of tumour progression [87] and, as a consequence, during the avascular stage, tumour 
development can be effectively controlled by effector cells : tumour-infiltrating cytotoxic lymphocytes (TICLs) [88]. The TICLs may be cytotoxic lymphocytes (CD8+ CTLs), natural killer-like (NK-like) cells and/or lymphokine activated killer (LAK) cells [89-92].

The effector cells work by combining with the tumor cells and destroy them by lysis (refers to the death of a cell by the breaking of the cellular membrane), that is,

$$
E+T \stackrel{k_{1}}{\longrightarrow} C \stackrel{k_{2}}{\longrightarrow} E+P
$$

where $C$ is a complex of effector and tumor cells, and $P$ is the product of lysis. The parameters $k_{1}$ and $k_{2}$ are non-negative kinetic constants : $k_{1}$ describe the rate of binding of TICLs to tumour cells without damaging cells; $k_{2}$ is the rate of detachment of TICLs from tumour cells, resulting in an irreversible programming of the tumour cells for lysis (i.e. death). Using the law of mass action, which states that the rate of a reaction is proportional to the product of the concentration of the reactants, neglecting the spatial variation of the cell of the immune system, the equations of the effector and the complex are given by

$$
\begin{aligned}
& \frac{d E}{d t}=-k_{1} E T+k_{2} C \\
& \frac{d C}{d t}=k_{1} E T-k_{2} C
\end{aligned}
$$

For the sake of simplicity, and since the generality of the problem is not lost, we make the assumption that lysis occurs much faster than the processes in the volume element considered (i.e. the time variations of the effector cells $E$ and of the complex of effector and tumor cells $C$ are much slower than the time variation of the product of lysis $P$ ) [105]. Thus we make the quasi-steady state hypothesis

$$
k_{1} E T-k_{2} C=0
$$

According to this assumption, by adding equations (21) and (22), we finally get

$$
\frac{d(E+C)}{d t}=0 \quad \text { which implies that } \quad E+C=E_{0}
$$

By substituting the value of $C$ into equation (23), we obtain 


$$
E=\frac{k_{2} E_{0}}{k_{1} T+k_{2}}
$$

Hence the rate at which $T$ is destroyed by immune cells is approximately given by

$$
-k_{1} E T=-\frac{k_{1} k_{2} E_{0} T}{k_{1} T+k_{2}}
$$

Thus, the equation for $T$, including the immune response, is given by

$$
\frac{\partial T}{\partial t}=\rho T\left(1-\frac{T}{K}\right)-\frac{k_{1} k_{2} E_{0} T}{k_{2}+k_{1} T}+D \nabla^{2} T
$$

Let

so we have

$$
f(T)=\rho T\left(1-\frac{T}{K}\right)-\frac{k_{1} k_{2} E_{0} T}{k_{2}+k_{1} T}
$$

$$
\frac{\partial T}{\partial t}=f(T)+D \nabla^{2} T
$$

Now, for small values of $T$, we can expand (26) in series, keeping the terms up to the second order and get

$$
f(T)=\rho T\left(1-\frac{T}{K}\right)-k_{1} E_{0} T\left(1-\frac{T}{k_{2} / k_{1}}\right)=\left(\rho-k_{1} E_{0}\right) T+\left(\frac{k_{1}^{2} E_{0}}{k_{2}}-\frac{\rho}{K}\right) T^{2}
$$

i.e. the immune response for small concentrations of the tumor cells $T$ presents also a logistic growth, this time $k_{1} E_{0}$ being the growth rate and $k_{2} / k_{1}$ the carrying capacity of the environment.

Let us see first, what happens with equation (29) with (30), if one considers the spacetime where the tumor cells $T$ move, changes from classical to non-differentiable.

We already know, according to Nottale [65], that a transition from classical (differentiable) mechanics to the scale relativistic framework is implemented by passing to a fluid-like description (the fractality of space), considering the velocity field a fractal function explicitly depending on a scale variable (the fractal geometry of each geodesic) and defining two fractal velocity fields which are fractal functions of the scale variable $d t$ (the non-differentiability of space). 
Consequently, replacing $d / d t$ by $\hat{d} / d t$ (using (17)) and solving for both real and imaginary parts, (29) with (30) becomes,

$$
\begin{aligned}
& \frac{\partial T}{\partial t}+\mathbf{V} \cdot \nabla T=f(T)+D \nabla^{2} T \\
& \mathbf{U} \cdot \nabla T+D \nabla^{2} T=0
\end{aligned}
$$

where $\underline{V}=\mathbf{V}-i \mathbf{U}$ is the complex velocity (we have identified the real velocity $\boldsymbol{V}$ with $\boldsymbol{v}$, the instantaneous velocity of the tumor cells) and $\mathscr{D}$ defines the "amplitude" of the fractal fluctuations.

We may notice a clear difference from the classical approach - the occurence (from the imaginary parts) of the second equation (31b), which is absolutely new.

Let us analyse in what follows, (31b) which, one can see, may contain some interesting biophysical features.

If we compare it with Navier-Stokes equation [93], where there are no pressure gradients, we can see the first term of (31b) gives the rate at which $T$ is transported through a 'fluid' by means of the motion of 'fluid' particles with the velocity $\boldsymbol{U}$; the second term gives the diffusion of $T,(\mathscr{D}$ which is the "amplitude" of the fractal fluctuations, plays here the role of the 'cinematic viscosity' of the 'fluid'). One can notice, in those regions in which the second term of (31b) is negligible, $D T / D t=0$. This means that in inviscid flows, for instance, $T$ is frozen into the 'particles of the fluid'. Physically this is because in an inviscid 'fluid' shear stresses are zero, so that there is no mechanism by which $T$ can be transferred from one 'fluid' particle to another. This may be the case for the transport of $T$ by $\boldsymbol{U}$ in the equation (31b).

If we consider the flow of $T$ induced by a uniform translatory motion of a plane spaced a distance $Y$ above a stationary parallel plane, and if the tumor cells concentration increases from zero (at the stationary plane) to $U$ (at the moving plane) like in the case of simple Couette flow, or simple shear flow, then

$$
\text { rate of shear deformation }=\frac{d T}{d y}=\frac{U}{Y}
$$


For many fluids it is found that the magnitude of the shearing stress is related to the rate of shear proportionally :

$$
T_{\text {shear }}=\eta \frac{d T}{d y}=\eta \frac{T}{Y}
$$

Fluids which obey (33) in the above situation are known as Newtonian fluids, which have a very small coefficient of viscosity. When such 'fluids' flow at reasonable velocities it is found that viscous effects appear only in thin layers on the surface of objects or surfaces over which the 'fluid' flows. That is, if one continues the analogy, and questions how might be $T$ transported by the motion of 'fluid' particles with the velocity $\boldsymbol{U}$, in equation $(31 \mathrm{~b})$, one can assume that the mechanism of transfer of $T$ from one particle of 'fluid' to another is achieved over small distances (in thin layers, as stated above).

To resume, the model considered here is the transport of $T$, along a small elementary distance $\Lambda$, by the 'particles' of a Newtonian fluid moving with velocity $\boldsymbol{U}$, where the stress tensor obeys (33), i.e.

$$
\frac{d T(x)}{d x}=\frac{T(x)}{\Lambda}
$$

like in the case of simple Couette flow, or simple shear flow.

Consequently, (31b) reduces to the scalar equation

$$
T^{\prime \prime}(x)+K^{2}(x) T(x)=0
$$

which is the time independent Schrödinger equation, and

$$
K^{2}(x)=\frac{1}{\Lambda D} U(x)
$$

with $\Lambda$ and $D$ having the significance of a small elementary distance and of the 'cinematic viscosity' (or "amplitude" of the fractal fluctuations), respectively, and $U(x)$ is the velocity of the 'Newtonian fluid', which is nothing but the imaginary part of the complex velocity [83].

For $D=\hbar / 2 m$ and small distances of the order of Compton length, $\Lambda=\hbar / m c$ [84], an equation similar to (35) was solved using the WKBJ approximation method in $[94,95]$. We 
derived there, for different shapes of the potential, both asimptotic solutions and quantization conditions $[94,95]$

$$
\int_{x_{1}}^{x_{2}} k d x^{\prime}=\left(n-\frac{1}{2}\right) \pi, \quad n=1,2,3, \ldots
$$

where $k$ is the wave number.

Consequently, in the case presented here we may find two ways of diffusion of the tumor cells : one normal diffusion described by eq. (31a) characterized by the diffusion parameter $D$ (random motility coefficient of the tumor cells), over distances of the order $\Lambda_{\mathrm{T}} \approx 200 \mu \mathrm{m}$ $[34,96,97]$ and a second diffusion over the Newtonian fluid, described by eq. (35) and characterized by $D$ the 'cinematic viscosity' (or "amplitude" of the fractal fluctuations), over much smaller distances of the order of Compton length, $\Lambda$ [84]. If Scale Relativity theory works, this might lead to a very important and new conclusion, that even in the avascular stages, a tumor might propagate and invade healthy tissues.

Furthermore, let us find if equation (31a) has a travelling wave solution. Consider a travelling wave solution of the form

$$
T(x, t)=\Phi(\xi), \quad \xi=x-c t
$$

Such a wave moves at a constant speed $c$ from left to right. So we have

$$
\begin{aligned}
& \frac{\partial T}{\partial t}=\frac{\partial \Phi}{\partial \xi} \frac{\partial \xi}{\partial t}=-\Phi^{\prime} c \\
& \frac{\partial T}{\partial x}=\frac{\partial \Phi}{\partial \xi} \frac{\partial \xi}{\partial x}=\Phi^{\prime} \\
& \frac{\partial^{2} T}{\partial x^{2}}=\Phi^{\prime \prime}
\end{aligned}
$$

where prime means derivative with respect to $\xi$.

By substituting (39) into equation (31a), we have

$$
-\Phi^{\prime} c+v \Phi^{\prime}=f(\Phi)+D \Phi^{\prime \prime}
$$

or with the substitutions 


$$
\begin{aligned}
& \alpha=\frac{1}{D}\left(\frac{\rho}{K}-\frac{k_{1}^{2} E_{0}}{k_{2}}\right) \\
& \beta=\frac{1}{D}\left(k_{1} E_{0}-\rho\right)
\end{aligned}
$$

we get

$$
\frac{d^{2} \Phi}{d \xi^{2}}+\frac{1}{D}(c-v) \frac{d \Phi}{d \xi}=\alpha \Phi^{2}+\beta \Phi
$$

If the convective processes are linear, i.e. $v=$ constant, equation (42a) with $M=v / c$, where $M$ is the Mach's number, becomes

$$
\frac{d^{2} \Phi}{d \xi^{2}}+\frac{c}{D}(1-M) \frac{d \Phi}{d \xi}=\alpha \Phi^{2}+\beta \Phi
$$

Moreover, if $M \approx 1$, equation (42b) takes the usual form

$$
\frac{d^{2} \Phi}{d \xi^{2}}=\alpha \Phi^{2}+\beta \Phi
$$

Equation (42c), although straightforward in appearance, is in fact rather difficult to solve because of the nonlinearity of the term $\Phi^{2}$. In order to obtain the exact solution of (42c), this equation is multiplied by $d \Phi / d \xi$, so it becomes

$$
\frac{d \Phi}{d \xi} \frac{d^{2} \Phi}{d \xi^{2}}=\alpha \Phi^{2} \frac{d \Phi}{d \xi}+\beta \Phi \frac{d \Phi}{d \xi}
$$

which can further be written as

$$
\frac{d}{d \xi}\left[\frac{1}{2}\left(\frac{d \Phi}{d \xi}\right)^{2}-\frac{\alpha}{3} \Phi^{3}-\frac{\beta}{2} \Phi^{2}-C_{0}\right]=0
$$

Equation (44) is immediately integrable and we get

$$
\pm \int \frac{d \Phi}{\sqrt{\Phi^{3}+\frac{3 \beta}{2 \alpha} \Phi^{2}+\frac{3 C_{0}}{\alpha}}}=\sqrt{\frac{2 \alpha}{3}}\left(\xi-\xi_{0}\right)
$$

Now, the third degree polinomial under the radical sign in (45) may be written as

$$
P(\Phi)=\Phi^{3}+\frac{3 \beta}{2 \alpha} \Phi^{2}+\frac{3 C_{0}}{\alpha}=\left(\Phi-\vartheta_{1}\right)\left(\Phi-\vartheta_{2}\right)\left(\Phi-\vartheta_{3}\right)
$$


where $\vartheta_{1} \leq \vartheta_{2} \leq \vartheta_{3} \in \Re$ are the roots of the cubics. Furthermore, if we make the substitution

$$
\Phi=\vartheta_{1}+\left(\vartheta_{2}-\vartheta_{1}\right) \sin ^{2} \varphi
$$

we get

$$
P(\Phi)=\Phi^{3}+\frac{3 \beta}{2 \alpha} \Phi^{2}+\frac{3 C_{0}}{\alpha}=\left(\vartheta_{3}-\vartheta_{1}\right)\left(\vartheta_{2}-\vartheta_{1}\right)^{2}\left(1-k^{2} \sin ^{2} \varphi\right) \sin ^{2} \varphi \cos ^{2} \varphi
$$

with

$$
k^{2}=\frac{\vartheta_{2}-\vartheta_{1}}{\vartheta_{3}-\vartheta_{1}}
$$

Using (48), taking into account (49) and the differential of (47), one may now re-write (45) in the form

$$
\pm \int \frac{d \varphi}{\sqrt{1-k^{2} \sin ^{2} \varphi}}=\sqrt{\frac{\alpha}{6}\left(\vartheta_{3}-\vartheta_{1}\right)}\left(\xi-\xi_{0}\right)
$$

One can see that it can be written as an elliptic integral, if we follow the standard procedure [98] $\sin \phi=u, \phi=\arcsin u$ and we find

$$
\pm \int \frac{d u}{\sqrt{1-u^{2}} \sqrt{1-k^{2} u^{2}}}=\sqrt{\frac{\alpha}{6}\left(\vartheta_{3}-\vartheta_{1}\right)}\left(\xi-\xi_{0}\right)
$$

or

$$
u=\operatorname{sn}\left[ \pm \gamma\left(\xi-\xi_{0}\right) ; k\right]
$$

where

$$
\gamma=\sqrt{\frac{\alpha\left(\vartheta_{3}-\vartheta_{1}\right)}{6}}
$$

Finally, after going back to the original variable we get the exact solution of (42c)

$$
\Phi=\vartheta_{1}+\left(\vartheta_{2}-\vartheta_{1}\right) \sin ^{2}\left\{\arcsin s n\left[ \pm \gamma\left(\xi-\xi_{0}\right) ; k\right]\right\}
$$

or after further straigthforward simplifications

$$
\Phi(\xi)=\vartheta_{2}-\left(\vartheta_{2}-\vartheta_{1}\right) c n^{2}\left[\gamma\left(\xi-\xi_{0}\right) ; k\right]
$$


with the modulus $k$ given by (49) and where we have dropped the minus sign from the argument of the $c n$ function since it is symmetric (see Fig. 5a for a plot of the solution (55)).

Now, let us find and analyse the solutions of the cubic (46) $\vartheta_{1}, \vartheta_{2}$ and $\vartheta_{3}$ first by reducing it to a monic trinomial. Substituting $\Phi$ by $\mathrm{Y}-\frac{\beta}{2 \alpha}$ (Tschirnhaus transformation) we get the equation

$$
\mathrm{Y}^{3}+p \mathrm{Y}+q=0
$$

where

$$
p=-\frac{3 \beta^{2}}{4 \alpha^{2}} ; \quad q=\frac{\beta^{3}}{4 \alpha^{3}}+\frac{3 C_{0}}{\alpha}
$$

For finding the roots, we use in what follows the trigonometric method. When a cubic equation has three real roots, the formulas expressing these roots in term of radicals involve complex numbers. A representation of these roots in term of cosine and arccosine allows to avoid complex numbers. The formulas which follow are true in general (except when $p=0$ ), but involve complex cosine and arccosine when there is only one real root.

Starting from equation (56), let us set $\mathrm{Y}=u \cos \theta$. The idea is to choose $u$ for identifying equation (56) with the identity

$$
4 \cos ^{3} \theta-3 \cos \theta-\cos (3 \theta)=0
$$

In fact, choosing $u=2 \sqrt{-\frac{p}{3}}$ and dividing equation (56) by $u^{3} / 4$ we get

$$
4 \cos ^{3} \theta-3 \cos \theta-\frac{3 q}{2 p} \sqrt{\frac{-3}{p}}=0
$$

Combining with above identity, we get

$$
\cos (3 \theta)=\frac{3 q}{2 p} \sqrt{\frac{-3}{p}}
$$

and thus the roots are 


$$
\mathrm{Y}_{l}=2 \sqrt{-\frac{p}{3}} \cos \left(\frac{1}{3} \arccos \left(\frac{3 q}{2 p} \sqrt{\frac{-3}{p}}\right)-l \frac{2 \pi}{3}\right) \quad \text { for } \quad l=0,1,2
$$

or getting back to the original variable and replacing $p$ and $q$ from (57) we get

$$
\vartheta_{k}=\frac{\beta}{\alpha} \cos \left[\frac{\pi}{3}(3-2 k)-\frac{1}{3} \arccos \left(1+\frac{12 \alpha^{2} C_{0}}{\beta^{3}}\right)\right]-\frac{\beta}{2 \alpha} \quad \text { for } k=1,2,3
$$

This formula is totally real if $p<0$ (which is always true, see (57)) and the argument of the arccosine is between -1 and 1 , which is equivalent to

$$
\frac{C_{0}}{\alpha^{2}}\left(\frac{\beta^{3}}{2 \alpha^{2}}+3 C_{0}\right) \leq 0
$$

which implies also $p<0$. Thus the above formula for the roots is totally real if and only if the three roots are real (as we assumed from the beginning). If $\frac{C_{0}}{\alpha^{2}}\left(\frac{\beta^{3}}{2 \alpha^{2}}+3 C_{0}\right)>0$ there is only one real root, the other two being complex conjugate.

Let us express the solution for two particular cases by taking the equal sign in the inequality (63), i.e. $C_{0}=0$ or $\beta^{3} / \alpha^{2}=-6 C_{0}$. The roots of the polinomial (46) according to (62) are

$$
\begin{aligned}
& \vartheta_{1}=\vartheta_{2}=0, \quad \vartheta_{3}=-\frac{3 \beta}{2 \alpha} \\
& \vartheta_{1}=\vartheta_{2}=-\frac{\beta}{\alpha}, \quad \vartheta_{3}=\frac{\beta}{2 \alpha}
\end{aligned}
$$

and the solution (55) with (53) and (49) writes

$$
\begin{aligned}
& \Phi(\xi)=\text { constant } \\
& \Phi(\xi)=-\frac{\beta}{\alpha}+\frac{3 \beta}{2 \alpha} \sec h^{2}\left(\frac{\sqrt{-\beta}}{2}\left(\xi-\xi_{0}\right)\right)
\end{aligned}
$$

respectively.

'Cancer dormancy' is an operational term used to describe the phenomenon of a prolonged quiescent state in which tumour cells are present, but tumour progression is not 
clinically apparent $[85,86,99]$. As a condition, cancer dormancy is often observed in breast cancers, neuroblastomas, melanomas, osteogenic sarcomas, and in several types of lymphomas, and is often found 'accidentally' in tissue samples of healthy individuals who have died suddenly $[100,101]$. In some cases, cancer dormancy has been found in cancer patients after several years of front-line therapy and clinical remission. The presence of these cancer cells in the body determines, finally, the outcome of the disease. In particular, age, stress factors, infections, act of treatment itself or other alterations in the host can provoke the initiation of uncontrolled growth of initially dormant cancer cells and subsequent waves of metastases [86,102]. Recently, some molecular targets for the induction of cancer dormancy and the re-growth of a dormant tumour have been identified $[103,104]$. However, the precise nature of the phenomenon remains poorly understood.

We see that, for a particular choice of parameters, we were able to explain the phenomenon of cancer dormancy (see the solution (66)). The behaviour of the cancer dormancy simulations can be described as highly irregular, depicting unstable and heterogeneous tumour cell distributions that are nonetheless characterized by a relatively low total number of tumour cells. This behaviour is consistent with several immunomorphological investigations with tumour spheroids infiltrated by TICLs.

Moreover, the alteration of certain parameters of the model resulted in the existence of a travelling-wave-like solution (see the solution (67)). These travelling waves are of great importance because when they exist, the tumour invades the healthy tissue at its full potential escaping the host's immune surveillance.

As a result the propagation of a perturbation in the tumor cell concentration $T$ is achieved by cnoidal modes (55) for the $M \approx 1$ regimes. These modes degenerate either in harmonic modes for $k \equiv 0$ and harmonic packet modes for $k \rightarrow 0$, or in solitons like (67) for $k \equiv 1$ and soliton packets for $k \rightarrow 1$. Practically one may distinguish between two distinct propagation regimes : i) non-quasi-autonomous structures like harmonic waves or harmonic packets of waves (see Fig. 5b 
for $k \rightarrow 0$ ); ii) quasi-autonomous structures like solitons or soliton packets (see Fig. 5c for $k \rightarrow$ 1). One notice that in the later situation, the quasi-structures conserve their "qualities".

\section{Conclusions}

In this paper we analysed a continuum model of cancer cell invasion of tissue which explicitly accounts for cytotoxic response of the immune system whereby the killer cells respond to the cancer cells, in the framework of the Scale Relativity Theory.

The main results of this work may be summarized as follows :

a) considering the space(-time) where tumor cells move, changes from classical to nondifferentiable, a second diffusion phenomena, over the Newtonian fluid, described by eq. (35) and characterized by $D$ the 'cinematic viscosity' (or "amplitude" of the fractal fluctuations), over much smaller distances of the order of Compton length, $\Lambda$ occurs - leading to a very important feature of malignant tumors, that even in avascular stages they might propagate and invade healthy tissues;

b) an analytical travelling wave solution (see eq. (55)) was established in this paper for a reduced system, which nonetheless captures the essential elements of the full model presented in $[105]$

c) for a particular choice of parameters, we were able to explain the phenomenon of cancer dormancy (see the solution (66)) - in order to see how much dependent are these results on the choice of parameters, in Appendix we perform a sensitivity analysis;

d) the propagation of a perturbation in the tumor cell concentration $T$ is achieved by cnoidal modes (55) for the $M \approx 1$ regimes - these modes degenerate either in harmonic modes for $k \equiv 0$ and harmonic packet modes for $k \rightarrow 0$, or in solitons like modes (67) for $k \equiv 1$ and soliton packets for $k \rightarrow 1$.

The ability of cancer cells to invade tissue is one of the defining characteristics of the disease. A more detailed knowledge of the processes involved in cancer invasion is therefore of 
the utmost importance for gaining a deeper understanding of tumour growth and development, and for the design of future anti-cancer strategies.

Systems biology has emerged during the last decade as a powerful new paradigm for research in the life sciences. It is based on the premise that the properties of complex systems consisting of many components that interact with each other in non-linear, nonadditive ways cannot be understood solely by focusing on the components. The system as a whole has emergent properties that are not visible at the parts level. It is clear that our modelling and analysis of a travelling-wave solution to a continuous mathematical model describing the growth of a solid tumour in the presence of an immune system response in the framework of Scale Relativity theory opens the gate for many other potential analysis and modelling of mechanisms of tumour-cell-host-cell interactions, complex kinetic reactions, intricate systems biology models based on space-time nondifferentiability, which proves to be the space-time where biological phenomena take place and in particular, where malignant tumors grow in.

\section{Appendix}

Let us reconsider the equation

$$
\frac{d^{2} \Phi}{d \xi^{2}}=\alpha \Phi^{2}+\beta \Phi
$$

By making the substitutions

$$
\begin{aligned}
& \frac{d \Phi}{d \xi}=\Phi^{\prime}=u \\
& \frac{d^{2} \Phi}{d \xi^{2}}=\Phi^{\prime \prime}=u^{\prime}
\end{aligned}
$$

equation (A1) becomes the system of equations

$$
\begin{aligned}
& \Phi^{\prime}=u \\
& u^{\prime}=\beta \Phi+\alpha \Phi^{2}
\end{aligned}
$$

In the linear approximation the stationary state of (A3) reads $u_{1}=\Phi_{1}=0$. Then, the characteristic equation reads 


$$
\left|\begin{array}{cc}
1-\lambda & 0 \\
0 & \beta-\lambda
\end{array}\right|=(1-\lambda)(\beta-\lambda)=0
$$

with the solutions

$$
\lambda_{1}=1, \quad \lambda_{2}=\beta
$$

Thus we have : i) if $\beta>0$ both eigenvalues are positive. The stationary state $(0,0)$ is an unstable node, so the phase trajectories diverge monotonically from the fixed point and often tend towards a new attractor; ii) if $\beta<0$, an eigenvalue is negative and the other one is positive. The stationary state $(0,0)$ is a saddle point. Along one direction of the phase space the trajectories converge and along another direction they diverge. As a result of this divergence, the stationary state $(0,0)$ is unstable and, in most cases, the trajectories tend to a new attractor.

By means of the transition from the unstable node to the saddle point, the behavior of the system in the phase space changes dramatically and one can expect to a bifurcation (for more details see [107]).

Now, as reads from $(55)$ - see Fig. 5a-c, it results that the "travelling waves" are "built of" cnoidal oscillating modes of the tumor cells concentration field. The modulus $k$ of the Jacobi elliptic function $c n$ implements various flowing regimes as follows : i) for $k \rightarrow 0$ the cnoidal modes degenerate in wave packets and for $k \equiv 0$ in harmonic waves; ii) for $k \rightarrow 1$ the cnoidal modes degenerate in soliton packets, and for $k \equiv 1$ in solitons - these sequences are specific to a flowing regime by means of cuasi-autonomous structures; iii) the transition between the two regimes occurs for $k \approx 0.7$, as can be found in [107].

Acknowledgments. We like to thank three anonymous referees for their constructive criticism of the manuscript. 


\section{References :}

[1] V. Kumar, A.K. Abbas, N. Fausto, Robbins and Cotran pathologic basis of disease, 7th ed., W.B. Saunders Co., 2004.

[2] JO’D McGee, P.G. Isaacson, N.A. Wright, editors., Oxford textbook of pathology, Oxford, Oxford University Press., 1992.

[3] Lud FJ van der Velden, Anneke L Francke, Lammert Hingstman and Dick L Willems, Dying from cancer or other chronic diseases in the Netherlands: ten-year trends derived from death certificate data, BMC Palliative Care 8:4, 2009.

[4] T. Lindahl, Instability and decay of the primary structure of DNA, Nature 362 (1993) 709715.

[5] B. Alberts, A. Johnson, J. Lewis, M. Raff, K. Roberts, P. Walter, Molecular biology of the cell, 4th ed. New York : Garland Science, 2002.

[6] H. Lodish, A. Berk, S.L. Zipurski, P. Matsudaira, D. Baltimore, J. Darnell, Molecular cell biology, 4th ed. New York: W.H. Freeman and Company, 2000.

[7] R. Araujo, D. McElwain, A history of the study of solid tumour growth : the contribution of mathematical modelling, Bull. Math. Biol. 66 (2004) 1039-1091.

[8] H.M. Byrne, T. Alarcon, M.R. Owen, S.D. Webb, P.K. Maini, Modelling aspects of cancer dynamics : a review, Phil. Trans. Roy. Soc. A 364 (2006) 1563-1578.

[9] R.M. Sutherland, Cell and environment interactions in tumour microregions : the multicell spheroid model, Science 240 (1988) 177-184.

[10] J.P. Freyer, P.L. Schor, Regrowth of cells from multicell tumour spheroids, Cell Tissue Kinet. 20 (1987) 249.

[11] A.D. Conger, M.C. Ziskin, Growth of mammalian multicellular tumour spheroids, Cancer Res. 43 (1983) 558-560.

[12] J.P. Freyer, R.M. Sutherland, Regulation of growth saturation and development of necrosis in EMT6VRO multicellular spheroids glucose and oxygen supply, Cancer Res. 46 (1986) 35043512.

[13] P.W. Vaupel, S. Frinak, H.I. Bicher, Heterogenous oxygen partial pressure and pH distribution in C3H mouse mammary adenocarcinoma, Cancer Res. 41 (1981) 2008-2013.

[14] J.P. Freyer, Role of necrosis in regulating the growth saturation of multicell spheroids, Cancer Res. 48 (1988) 2432-2439.

[15] J. Landry, J.P. Freyer, R.M. Sutherland, Shedding of mitotic cells from the surface of multicell spheroids during growth, J. Cell. Physiol. 106 (1981) 23-32.

[16] J. Folkman, M. Hochberg, Self-regulation of growth in three dimensions, Exp. Med. 138 (1973) 745-753.

[17] M. Haji-Karim, J. Carlsson, Proliferation and viability in cellular spheroids of human origin, Cancer Res. 38 (1978) 1457-1464.

[18] W.R. Inch, J.A. McCredie, R.M. Sutherland, Growth of modular carcinomas in rodents compared with multicell spheroids in tissue culture, Growth 34 (1970) 271-282.

[19] J. Folkman, Tumour angiogenesis : therapeutic implications, New Engl. J. Med. 285 (1971) $1182-1186$.

[20] J. Adam, General aspects of modeling tumor growth and the immune response, in : J. Adam, N. Bellomo(eds), A Survey of Models on Tumor Immune Systems Dynamics, Birkhauser, Boston, MA (1996).

[21] N. Bellomo, E. de Angelis, L. Preziosi, Multiscale modelling and mathematical problems related to tumor evolution and medical therapy, J. Theor. Med. 5 (2003) 111-136.

[22] V. Quaranta, A.M. Weaver, P.T. Cummings, A.R.A. Anderson, Mathematical modeling of cancer : the future of prognosis and treatment, Clin. Chim. Acta 357 (2005) 173-179.

[23] S. Sanga, J.P. Sinek, H.B. Frieboes, J.P. Fruehauf, V. Cristini, Mathematical modeling of cancer progression and response to chemotherapy, Expert. Rev. Anticancer Ther. 6 (2006) 13611376. 
[24] T. Alarcón, H.M. Byrne, P.K. Maini, A cellular automaton model for tumour growth in inhomogeneous environment, J. Theor. Biol. 225 (2003) 257-274.

[25] A.R.A. Anderson, A hybrid mathematical model of solid tumour invasion: the importance of cell adhesion, IMA Math. Appl. Med. Biol. 22 (2005) 163-186.

[26] D.G. Mallett, L.G. de Pillis, A cellular automata model of tumor immune system interactions, J. Theor. Biol. 239 (2006) 334-350.

[27] R.G. Abbott, S. Forrest, K.J. Pienta, Simulating the hallmarks of cancer, Artif. Life 12 (2006) 617- 634.

[28] Y. Mansury, M. Kimura, J. Lobo, T.S. Deisboeck, Emerging patterns in tumor systems : simulating the dynamics of multicellular clusters with an agent-based spatial agglomeration model, J. Theor. Biol. 219 (2002) 343-370.

[29] H.M. Byrne, M.A.J. Chaplain, Growth of necrotic tumors in the presence and absence of inhibitors, Math. Biosci. 135 (1996) 187-216.

[30] H.M. Byrne, M.A.J. Chaplain, Modelling the role of cell-cell adhesion in the growth and development of carcinomas, Math. Comput. Model. 24 (1996) 1-17.

[31] H.P. Greenspan, On the growth and stability of cell cultures and solid tumors, J. Theor. Biol. 56 (1976) 229-242.

[32] H.M. Byrne, P. Matthews, Asymmetric growth of models of avascular solid tumors: exploiting symmetries, IMA J. Math. Appl. Med. Biol. 19 (2002) 1-29.

[33] M.A.J. Chaplain, M. Ganesh, I.G. Graham, Spatio-temporal pattern formation on spherical surfaces: numerical simulation and application to solid tumour growth, J. Math. Biol. 42 (2001) 387-423.

[34] V. Cristini, J.S. Lowengrub, Q. Nie, Nonlinear simulation of tumor growth, J. Math. Biol. 46 (2003) 191-224.

[35] X. Li, V. Cristini, Q. Nie, J.S. Lowengrub, Nonlinear three dimensional simulation of solid tumor growth, Disc. Cont. Dyn. Sys. B 7 (2007) 581-604 .

[36] D. Ambrosi D, F. Guana, Stress-modulated growth, Math. Mech. Solids. (in press) doi:10.1177/1081286505059739

[37] D. Ambrosi, F. Mollica, On the mechanics of a growing tumor, Int. J. Eng. Sci. 40 (2002) 1297-1316.

[38] D. Ambrosi, L. Preziosi, On the closure of mass balance models for tumor growth, Math. Mod. Meth. Appl. Sci. 12 (2002) 737-754.

[39] R.P. Araujo, D.L.S McElwain, A linear-elastic model of anisotropic tumor growth, Eur. J. Appl. Math. 15 (2004) 365-384.

[40] R.P. Araujo, D.L.S. McElwain, A mixture theory for the genesis of residual stresses in growing tissues II : solutions to the biphasic equations for a multicell spheroid, SIAM J. Appl. Math. 66 (2005) 447-467.

[41] A.F. Jones, H.M. Byrne, J.S. Gibson, J.W. Dold, A mathematical model of the stress induced during avascular tumor growth, J. Math. Biol. 40 (2000) 473-499.

[42] T. Roose, P.A. Netti, L.L. Munn, Y. Boucher, R. Jain, Solid stress generated by spheroid growth estimated using a linear poroelastic model, Microvasc. Res. 66 (2003) 204-212.

[43] H. Byrne, L. Preziosi, Modelling solid tumour growth using the theory of mixtures, Math. Med. Biol. 20 (2003) 341-366.

[44] M.A.J. Chaplain, L. Graziano, L. Preziosi, Mathematical modelling of the loss of tissue compression responsiveness and its role in solid tumour development, Math. Med. Biol. 23 (2006) 192-229.

[45] X. Zheng, S.M. Wise, V. Cristini, Nonlinear simulation of tumor necrosis, neovascularization and tissue invasion via an adaptive finite element/level set method, Bull. Math. Biol. 67 (2005) 211-259.

[46] A.R.A. Anderson, M.A.J. Chaplain, Continuous and discrete mathematical models of tumor-induced angiogenesis, Bull. Math. Biol. 60 (1998) 857-900. 
[47] J. Sinek, H. Frieboes, X. Zheng, V. Cristini, Two-dimensional chemotherapy simulations demonstrate fundamental transport and tumor response limitations involving nanoparticles, Biomed. Microdev. 6 (2004) 197-309.

[48] V. Cristini, H.B. Frieboes, R. Gatenby, S. Caserta, M. Ferrari, J. Sinek, Morphological instability and cancer invasion, Clin. Cancer Res. 11 (2005) 6772-6779.

[49] H.B. Frieboes, X. Zheng, C-H. Sun, B. Tromberg, R. Gatenby, V. Cristini, An integrated computational/experimental model of tumor invasion, Cancer Res. 66 (2006) 1597-1604.

[50] C.S. Hogea, B.T. Murray, J.A. Sethian, Simulating complex tumor dynamics from avascular to vascular growth using a general level-set method, J. Math. Biol. 53 (2006) 86-134 .

[51] P. Macklin, J. Lowengrub, A new ghost cell/level set method for moving boundary problems: application to tumor growth, J. Scientific Comp. (in review).

[52] P. Macklin, J. Lowengrub, Nonlinear simulation of the effect of microenvironment on tumor growth, J. Theor. Biol. 245 (2007) 677-704.

[53] S.M. Wise, J.S. Lowengrub, H.B. Frieboes, V. Cristini, Three dimensional diffuse-interface simulation of multispecies tumor growth- I : numerical method, Bull. Math. Biol. (in review)

[54] H.B. Frieboes, S.M. Wise, J.S. Lowengrub, V. Cristini, Three dimensional diffuse-interface simulation of multispecies tumor growth-II : investigation of tumor invasion, Bull. Math. Biol. (in review)

[55] H.B. Frieboes, J.S. Lowengrub, S. Wise, X. Zheng, P. Macklin, E.L. Bearer, V. Cristini, Computer simulation of glioma growth and morphology, NeuroImage 37 (2007) S59-S70.

[56] P. Waliszewski, M. Molski, J. Konarski, On the holistic approach in cellular and cancer biology : nonlinearity, complexity and quasideterminism of dynamic cellular network, J Surg Oncol 68 (1998) 70-80.

[57] P. Waliszewski, M. Molski, J. Konarski, Self-similarity, collectivity and evolution of fractal dynamics during retinoid-induced differentiation of cancer cell population, Fractals 7 (1999) $139-149$.

[58] P. Waliszewski, J. Konarski, M. Molski, On the modification of fractal self-space during cell differentiation or tumor progression, Fractals 8 (2000) 195-203.

[59] P. Waliszewski, M. Molski, J. Konarski, On the relationship between fractal geometry of space and time in which a cellular system exists and dynamics of gene expression, Acta Biochim Pol 48 (2001) 209-220.

[60] V.V. Isaeva, E.V. Puschina, Y.A. Karetin, The quasi-fractal structure of fish brain neurons, Russ J Mar Biol 30 (2004) 127-134.

[61] J. Pastor, A. Bru, I. Fernaud, C. Berenguer, M.J. Fernaud, S. Melle, Fractal dimension and temporal evolution of neurons, An Fis (RSEF) 10 (1998) 1-2.

[62] A. Einstein, Die Grundlage der allgemeinen Relativitätstheorie, Ann. Phys. 49 (1916) 769822.

[63] L. Nottale, Fractals and the quantum theory of spacetime, Int. J. Mod. Phys. A 4 (1989) 5047-5117.

[64] L. Nottale, The theory of scale relativity, Int. J. Mod. Phys. A 7 (1992) 4899-4936.

[65] L. Nottale, Fractal Space-Time and Microphysics : Towards a Theory of Scale Relativity, World Scientific, Singapore, 1993.

[66] C. Auffray, L. Nottale, Scale relativity theory and integrative systems biology : 1 Founding principles and scale laws, Progress in Biophysics and Molecular Biology 97 (2008) 79-114.

[67] L. Nottale, Scale relativity and fractal space-time: applications to quantum physics, cosmology and chaotic systems, Chaos Solitons Fractals 7 (1996) 877-938.

[68] R.P. Feynman, A.R. Hibbs, Quantum Mechanics and Path Integrals, MacGraw-Hill, New York, NY, 1965.

[69] B. Mandelbrot, Les Objets Fractals, Flammarion, Paris, 1975.

[70] L.F. Abbott, M.B. Wise, Dimension of a quantum-mechanical path, Am. J. Phys. 49 (1981) $37-39$. 
[71] L. Nottale, J. Schneider, Fractals and nonstandard analysis, J. Math. Phys. 25 (1984) 12961300 .

[72] G.N. Ord, Fractal space-time - a geometric analog of relativistic quantum mechanics, J. Phys. A Math. Gen. 16 (1983) 1869-1884.

[73] J. Cresson, Quelques problèmes de physique mathématique, Habilitation à diriger des Recherches, Franche-Comté University, Besançon, France, 2001.

[74] J. Cresson, Scale calculus and the Schrödinger equation, J. Math. Phys. 44 (2003) 49074938.

[75] M.N. Célérier, L. Nottale, Quantum-classical transition in scale relativity, J. Phys. A : Math. Gen. 37 (2004) 931-955.

[76] L. Nottale, Scale relativity-from quantum mechanics to chaotic dynamics, Chaos Solitons Fractals 6 (1995) 399-410.

[77] L. Nottale, Scale-relativity and quantization of extra-solar planetary systems, Astron. Astrophys. Lett. 315 (1996) L9-L12.

[78] L. Landau, E. Lifchitz, Fluid Mechanics, Mir, Pergamon, Moscow, Russia, New York, NY, 1959.

[79] G.N. Ord, A.S. Deakin, Random walks, continuum limits, and Schrödinger's equation, Phys. Rev. A 54 (1996) 3772-3778.

[80] G.N. Ord, J.A. Galtieri, The Feynman propagator from a single path, Phys. Rev. Lett. 89 (2002) 250403.

[81] G. Jumarie, Fractionalization of the complex-valued Brownian motion of order $n$ using Riemann-Liouville derivative. Applications to mathematical finance and stochastic mechanics, Chaos Solitons Fractals 5 (2006) 1285-1305.

[82] L. Nottale, Origin of complex and quaternionic wavefunctions in quantum mechanics : the scale-relativistic view. in : Angle`s, P. (Ed.), Proceedings of the Seventh International Colloquium on Clifford Algebra. Birkhäuser Verlag, Springer, New York, NY, 2008.

[83] J.C. Pissondes, Quadratic relativistic invariant and metric form in quantum mechanics, J. Phys. A : Math. Gen. 32 (1999) 2871-2885.

[84] L. Nottale, The theory of scale relativity: non-differentiable geometry and fractal spacetime. in: American Institute of Physics Conference Proceedings, vol. 718 , 2004, pp. 68-95.

[85] V. Schirrmacher, T-cell immunity in the induction and maintenance of a tumour dormant state, Semin. Cancer Biol. 11 (2001) 285-295.

[86] J.W. Uhr, R. Marches, Dormancy in a model of murine B cell lymphoma, Semin. Cancer Biol. 11 (2001) 277-283.

[87] P.G. Coulie, Human tumor antigens recognized by T cells: new perspectives for anti-cancer vaccines ?, Mol. Med. Today 3 (1997) 261-268.

[88] D. Loeffler, S. Ratner, In vivo localization of lymphocytes labeled with low concentrations of HOECHST-33342, J. Immunol. Methods 119 (1989) 95-101.

[89] R.A. Deweger, B. Wilbrink, R.M.P. Moberts, D. Mans, R. Oskam, W. den Otten, Immune reactivity in SL2 lymphomabearing mice compared with SL2-immunized mice, Cancer Immunol. Immunother. 24 (1987) 1191-1192.

[90] G. Forni, G. Parmiani, A. Guarini, R. Foa, Gene transfer in tumour therapy, Ann. Oncol. 5 (1994) 789-794.

[91] E.M. Lord, G. Burkhardt, Assessment of in situ host immunity to syngeneic tumours utilizing the multicellular spheroid model, Cell. Immunol. 85 (1984) 340-350.

[92] K.M. Wilson, E.M. Lord, Specific (EMT6) and non-specific (WEHI-164) cytolytic activity by host cells infiltrating tumour spheroids, Br. J. Cancer 55 (1987) 141-146.

[93] P.D. McCormack, L. Crane, Physical Fluid Mechanics, London - NewYork: Academic Press., 1973.

[94] C.Gh. Buzea, C. Bejinariu, C. Boris, P. Vizureanu, M. Agop, Motion of free particles in fractal space-time, Intern. J. of Nonl. Sci. and Num. Sim. 10(11-12) (2009) 1399-1414.

[95] C.Gh. Buzea, I. Rusu, V. Bulancea, G. Badarau, V.P. Paun, M. Agop, The Time Dependent 
Ginzburg-Landau Equation in Fractal Space-Time (II), Physics Letters A 374 (2010) 27572765.

[96] P. Macklin, Numerical simulation of tumor growth and chemotherapy, Master's Thesis, University of Minnesota School of Mathematics, 2003.

[97] P. Macklin, J.S. Lowengrub, Evolving interfaces via gradients of geometry-dependent interior Poisson problems : application to tumor growth, J. Comput. Phys. 203 (1) (2005) 191220.

[98] F. Bowman, Introduction to elliptic functions with applications, London : English Universities Press Ltd., 1953.

[99] E. Yefenof, Cancer dormancy: from observation to investigation and onto clinical intervention, Semin. Cancer Biol. 11 (2001) 269-270.

[100] A. Alsabti, Tumour dormant state, Tumour Res. 13 (1) (1978) 1-13.

[101] N. Breslow, C.W. Chan, G. Dhom, R.A. Drury, L.M. Franks, B. Gellei, Y.S. Lee, S. Lundberg, B. Sparke, N.H. Sternby, H. Tulinius, Latent carcinoma of prostate at autopsy in seven areas, Int. J. Cancer 20 (5) (1977) 680-688.

[102] L. Holmberg, M. Baum, Work on your theories !, Nat. Med. 2 (8) (1996) 844-846.

[103] J.A. Aguirre Ghiso, Inhibition of FAK signaling activated by urokinase receptor induces dormancy in human carcinoma cells in vivo, Oncogene 21 (16) (2002) 2513-2524.

[104] T. Udagawa, A. Fernandez, E.G. Achilles, J. Folkman, R.J. D'Amato, Persistence of microscopic human cancers in mice: alterations in the angiogenic balance accompanies loss of tumor dormancy, FASEB J. 16 (11) (2002) 1361-1370.

[105] A. Matzavinos, M.A.J. Chaplain, Travelling-wave analysis of a model of the immune response to cancer, C. R. Biologies 327 (2004) 995-1008.

[106] M.L. Martins, S.C. Ferreira Jr., M.J. Vilela, Multiscale models for the growth of avascular tumors, Physics of Life Reviews 4 (2007) 128-156.

[107] E.A. Jackson, Perspectives in nonlinear dynamics, Cambridge University Press, Cambridge, London, 1991. 


\section{Figure captions}

Fig. 1. Reactive oxygen species, (by)products of normal cell aerobic metabolism and of lipid peroxidation, as well as other small reactive molecules promote hydrolytic attack to, oxidative damage in, and nonenzymatic methylation of DNA. In (a) the target sites for these spontaneous reactions in one strand of the DNA double helix are shown. (b) Hydrolytic depurination generates apurinic sites in DNA and, in consequence, a loss of genetic information. (c) Hydrolytic deamination of cytosine to uracil and (d) oxidation of guanine residues to 8hydroxyguanine in DNA are the two major spontaneous premutagenic events in living cells. (e) Mutations produced after replication by such chemical alterations of the nucleotides (adapted from [106]).

Fig. 2. Many and varied external carcinogens trigger cancer by creating mutations on DNA. Therefore, they are really mutagens. For instance, (a) a single point mutation in normal human ras gene (five thousand DNA bases long) transforms it into the bladder carcinoma oncogene. This minimal change can be the result of an unrepaired oxidation of guanine to 8hydroxyguanine, which pairs preferentially with adenine rather than cytosine. (b) Exposure to ultraviolet light causes formation of dimers between two successive pyrimidinic bases (cytosine or thymine) which induce copying errors. (c) Uncorrected rejoining of chromosomal breaks caused by, for instance X-rays or radiation, can juxtapose DNA segments that were previously in different chromosomes (translocations). In Burkitt's lymphoma, a normal myc proto-oncogene becomes improperly fused to sequences regulating the expression of antibodies in B cells. The translocated myc gene, now regulated by the antibody enhancer, is persistently expressed and drives the excessive proliferation of the mutated B cells. This kind of mutation explains why cancers of the immune system are the most frequent in people exposed to radiation (adapted from [106]).

Fig. 3 Dependence on the time-scale, $\ln (d t / T)$, of the logarithm of the velocity $\ln \left(V / V_{0}\right)$ on a fractal geodesic, including a classical (differentiable) part, which is dominant at large scale (it tends to a scale-independent velocity toward the right in the figure), and a fractal (nondifferentiable) fluctuating part, which is dominant at small scales (to the left). The fluctuation has been taken here to be Gaussian. The fluctuating fractal part $d \xi$ is of order $d t^{1 / 2}$ for fractal dimension 2 , so that the velocity diverges toward small scales as $d t^{-1 / 2}$, which expresses the nondifferentiability of the fractal coordinate.

Fig. 4 A fractal function. An example of such a fractal function is given by the projections of a fractal curve on Cartesian coordinates, in function of a continuous and monotonous parameter (here the time $t$ ) which marks the position on the curve. The figure also exhibits the relation between space and time differential elements for such a fractal function, and compares the differentiable and non-differentiable parts of the space elementary displacement. While the "classical" coordinate variation $\delta x=\langle\delta X>$ is of the same order as the time differential $\delta t$, the fractal fluctuation becomes much larger than $\delta t$ when $\delta t<<T$, where $T$ is a transition time scale, and it depends on the fractal dimension $D_{\mathrm{F}}$ as : $\delta \xi \propto \delta t^{1 / D_{F}}$. Therefore the two contributions to the full differential displacement are related by the fractal law $\delta \xi^{D_{F}} \propto \delta x$, since $\delta x$ and $\delta t$ are differential elements of the same order.

Fig. 5 a) cnoidal modes of propagation of a perturbation in the tumor cells concentration $T$; b) harmonic modes of propagation for $k=0$; c) solitonic modes of propagation for $k \equiv 1$. 

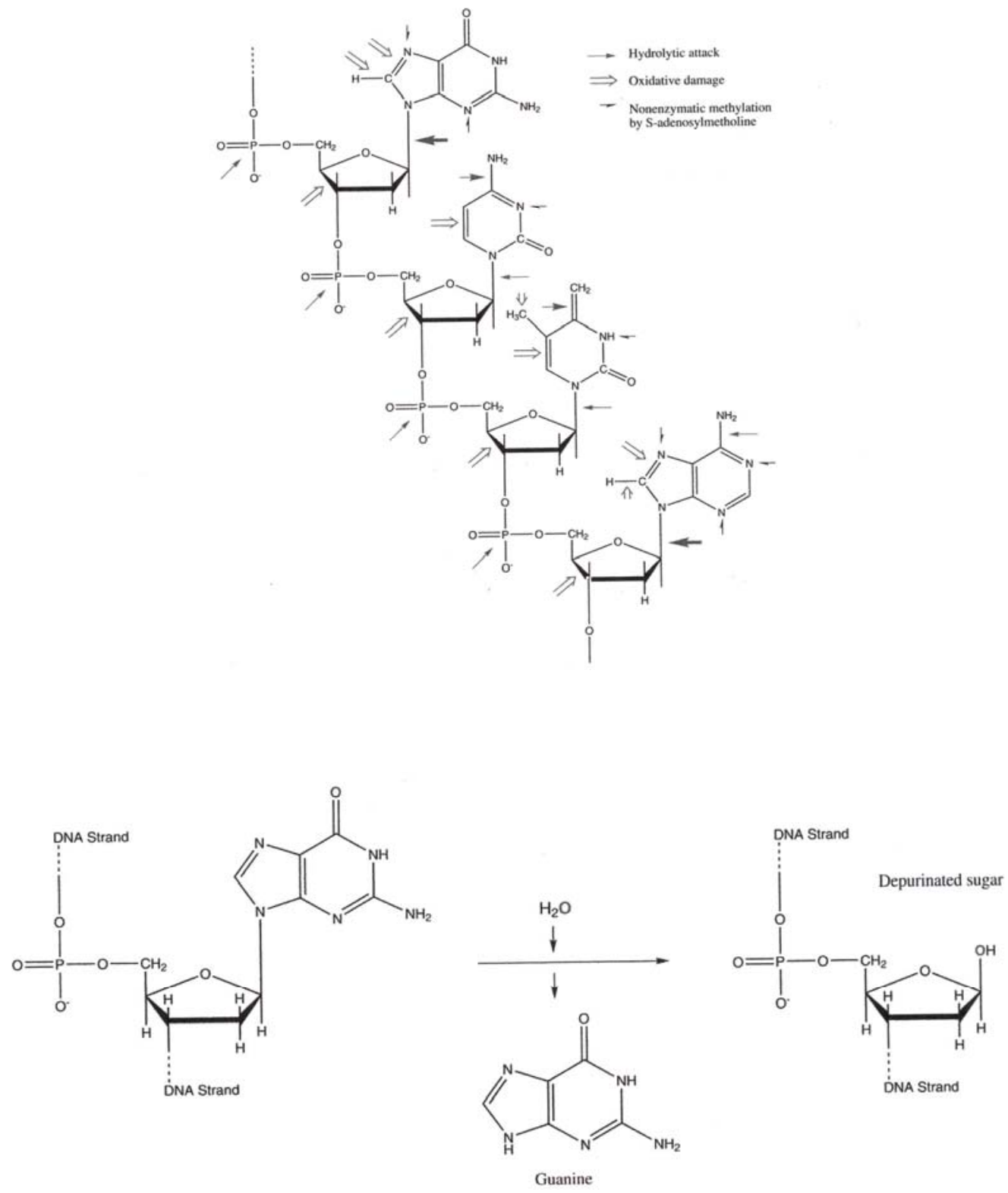


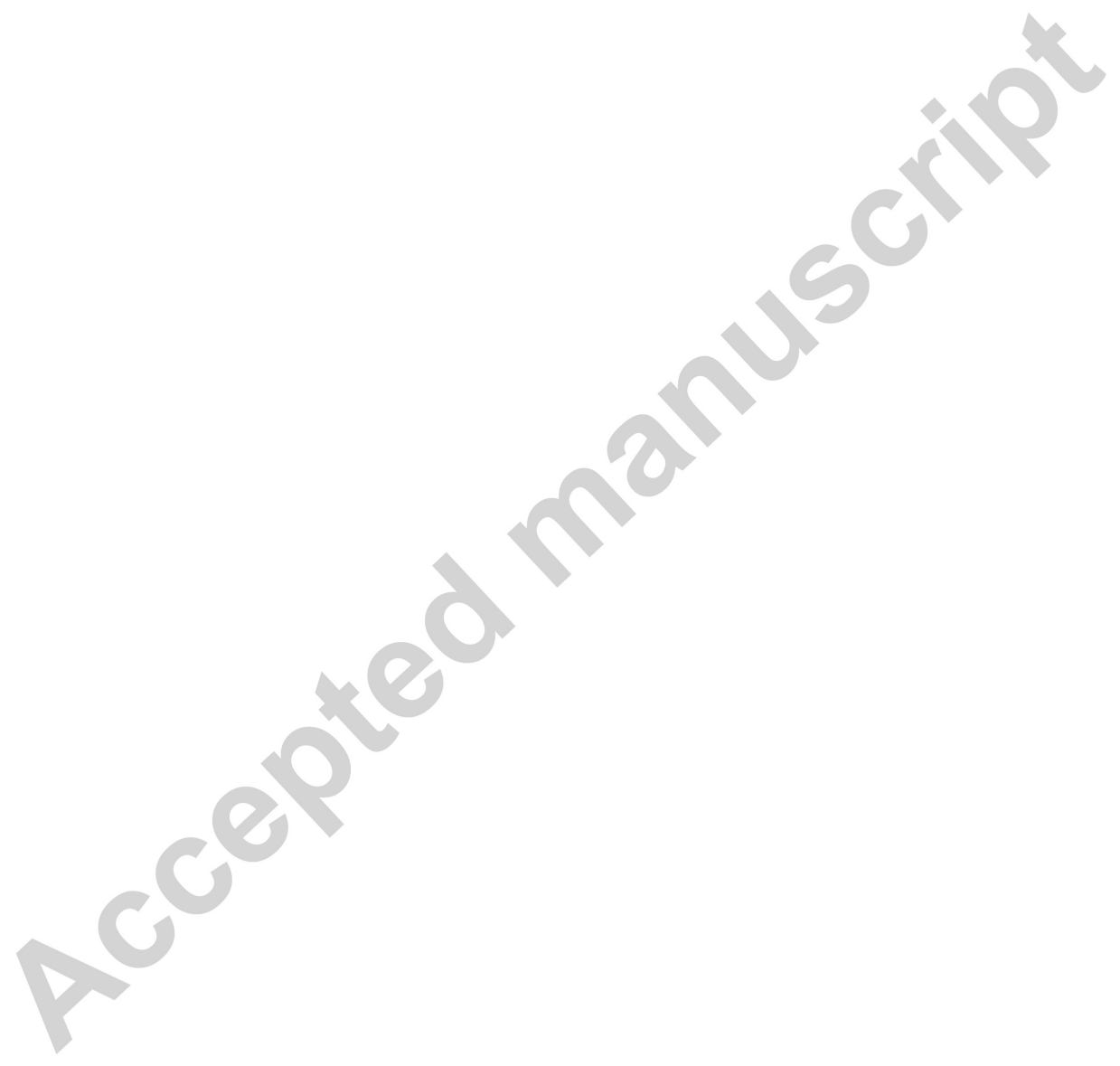


<smiles>Nc1ccn(C2OC[C@H](COP(=O)(O)OI)[C@@H](I)[C@@H]2I)c(=O)n1</smiles>

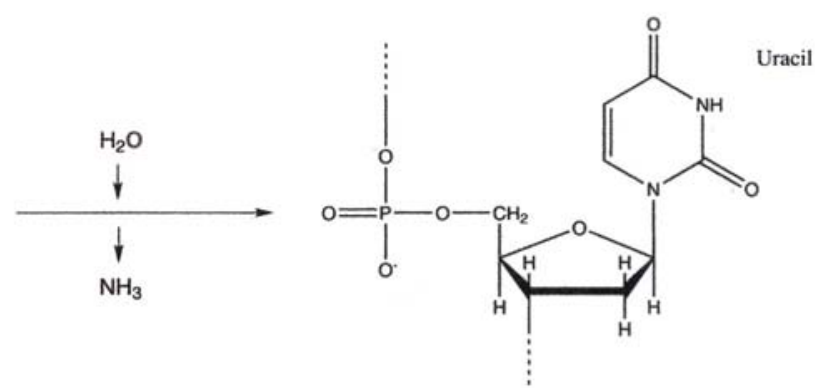<smiles></smiles><smiles>Nc1nc2[nH]c(O)nc2c(=O)[nH]1</smiles>

a $\mathrm{G}$ changed by an $\mathrm{A}$

\begin{tabular}{c|c|c|c} 
& & \multicolumn{2}{c}{$\begin{array}{c}\text { deaminated } \\
\text { carbon }\end{array}$} \\
\hline & \multicolumn{1}{c}{} & \\
\hline $\mathrm{T}$ & $\mathrm{U}$ & $\mathrm{A}$ & $\mathrm{T}$ \\
$\mathrm{A}$ & $\mathrm{G}$ & $\mathrm{T}$ & $\mathrm{A}$ \\
\hline
\end{tabular}

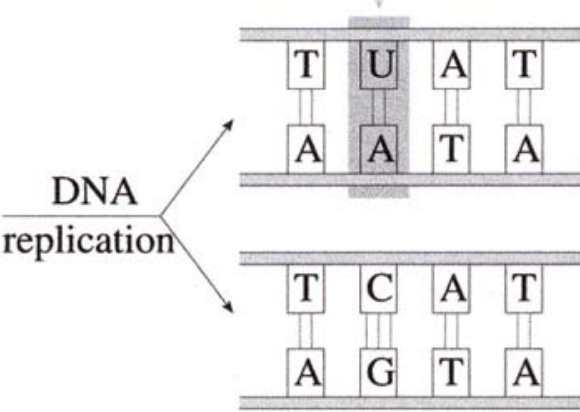

original strand

mutated DNA

(point mutation)

new strand

new strand

unchanged DNA

original strand

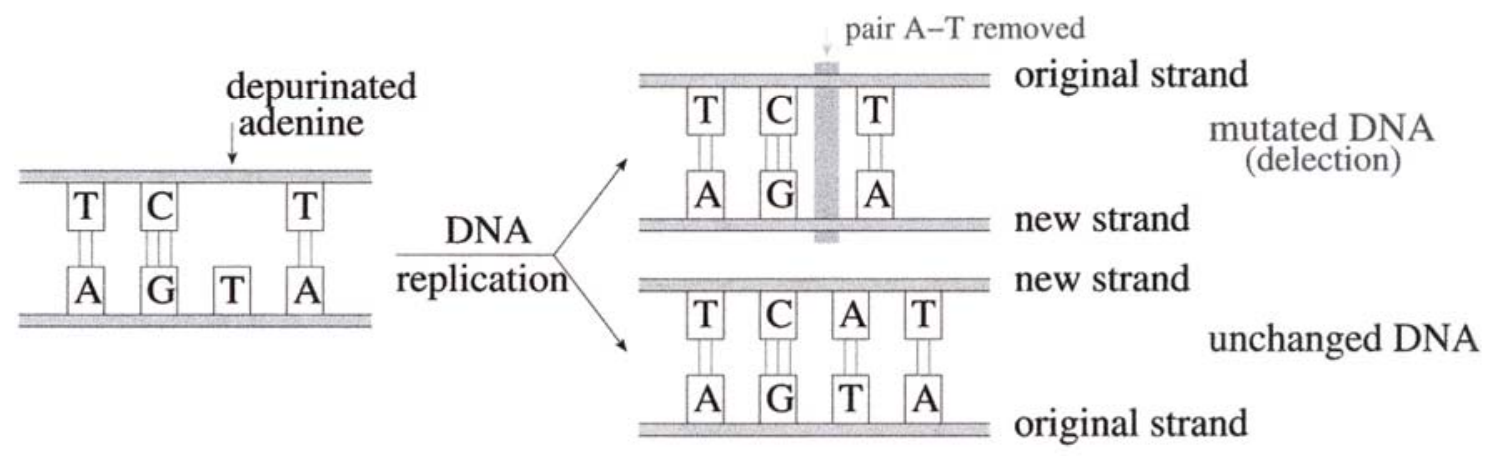


CCCGGG CCGCAGGCCC TTGAGGAGCG

gly $\longrightarrow$ proto-oncogene

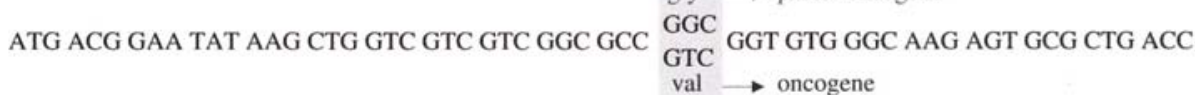
ATC CAG CTG ATC CAG AAC CAT TTT GTG GAC GAA TAC GAC CCC ACT ATA GAG GTGAGCCTGC

GCCGCCGTCC AGGTGCCAGC AGCTGCTGCG GGCGAGCCCA GGACAGCC AGGATAGGGCTGGCTGCAGC CCCTGGTCCC CTGCATGGTG CTGTGGCCCT GTCTCCTGCT TCCTCTAGAG GAGGGGAGTC CCTCGTCTCA GCACCCCAGG AGAGGAGGGG GCATGAGGGG CATGAGAGGT ACC
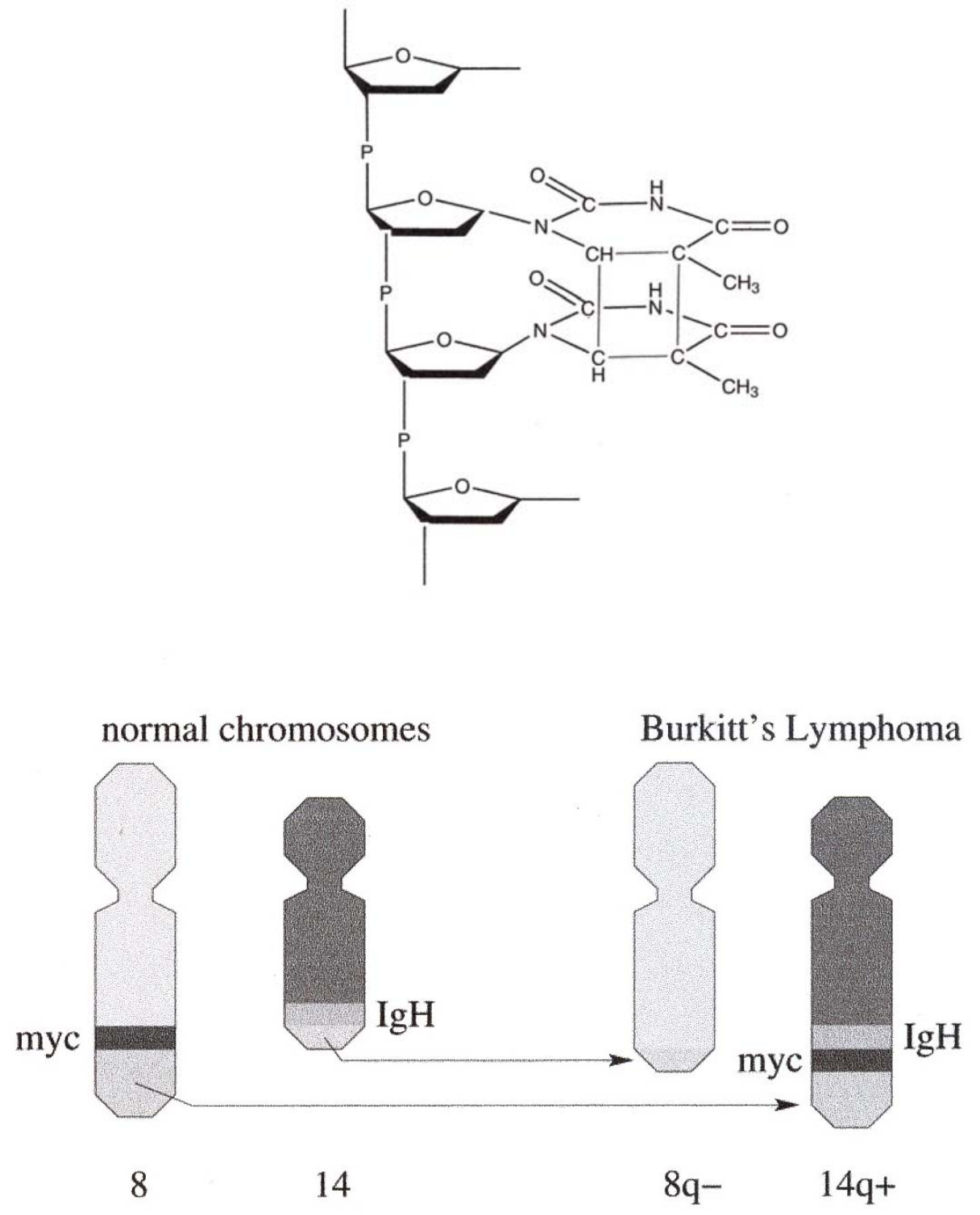

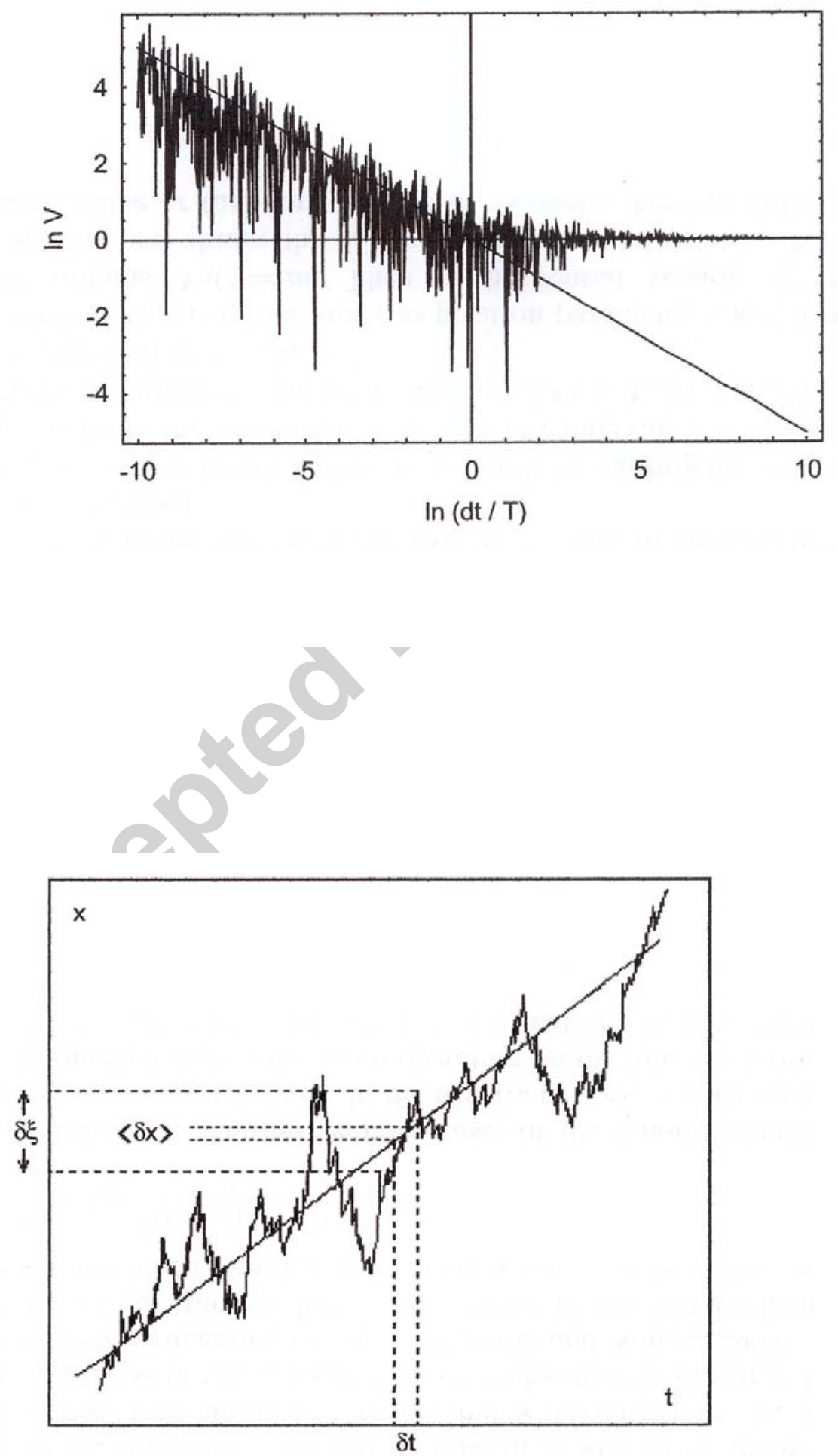

Fig. $4 \quad 42$ 

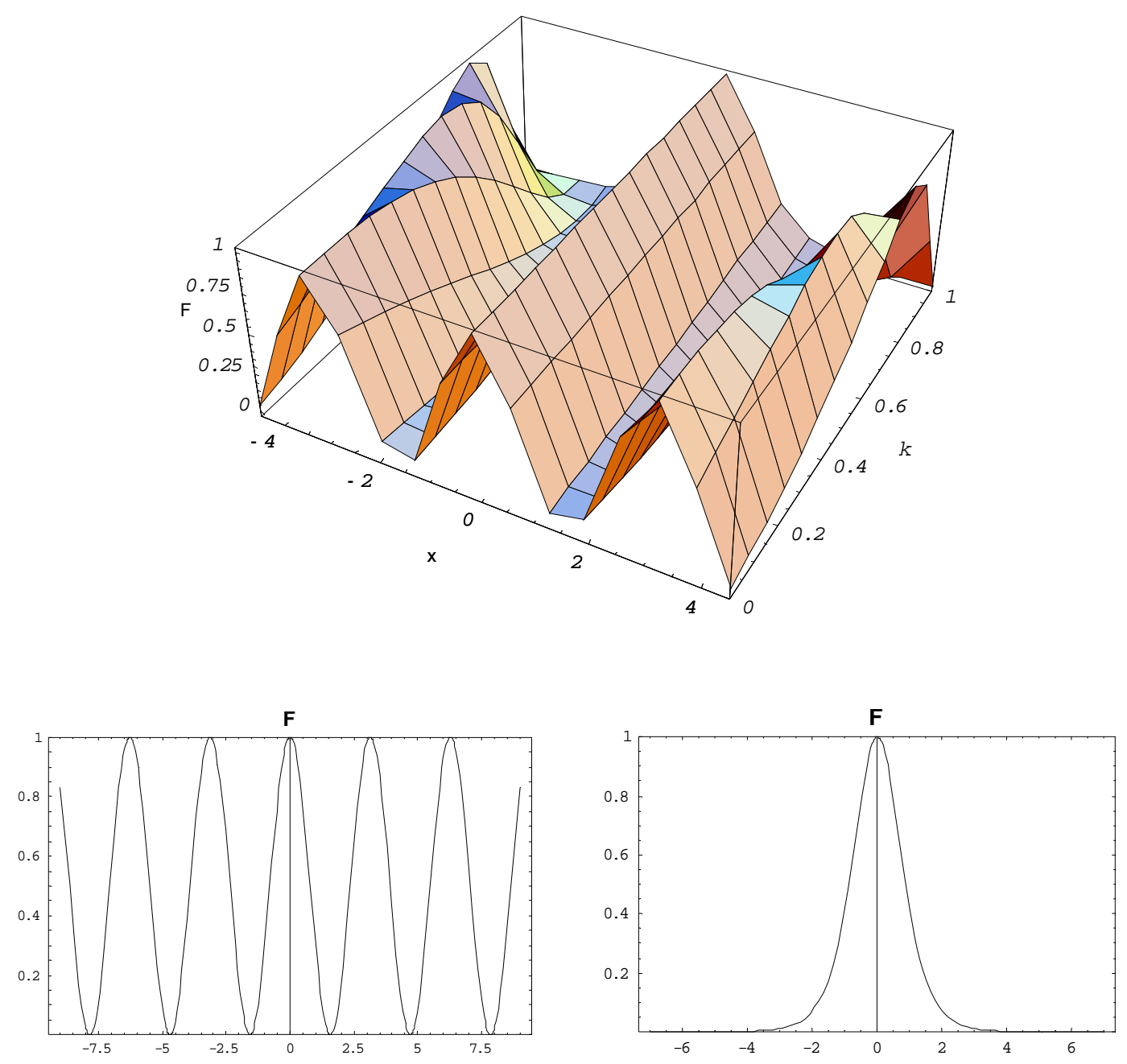